\title{
Endpoint resummation in squark decays
}

\author{
Chul Kim, ${ }^{1}$ Jeong Han Kim $\odot,{ }^{2,3}$ Seung J. Lee, ${ }^{4}$ and Jure Zupan ${ }^{5}$ \\ ${ }^{1}$ Institute of Convergence Fundamental Studies and School of Liberal Arts, \\ Seoul National University of Science and Technology, Seoul 01811, Korea \\ ${ }^{2}$ Department of Physics, University of Notre Dame, Notre Dame, Indiana 46556, USA \\ ${ }^{3}$ Department of Physics and Astronomy, University of Kansas, Lawrence, Kansas 66045, USA \\ ${ }^{4}$ Department of Physics, Korea University, Seoul 136-713, Korea and School of Physics, \\ Korea Institute for Advanced Study, Seoul 130-722, Korea \\ ${ }^{5}$ Department of Physics, University of Cincinnati, Cincinnati, Ohio 45221, USA
}

(Received 4 December 2018; revised manuscript received 12 October 2019; accepted 28 May 2020; published 11 June 2020)

\begin{abstract}
We study soft and collinear gluon emission in squark decays to quark-neutralino pair, at next-to-next-toleading logarithmic (NNLL) accuracy in the end-point region, using soft collinear effective theory (SCET), and at next-to-leading (NLO) fixed order in the rest of the phase space. As a phenomenological case study we discuss the impact of radiative corrections on the simultaneous measurements of squark and neutralino masses at a linear $e^{+} e^{-}$collider based on $\sqrt{s}=3 \mathrm{TeV}$ Compact Linear Collider (CLIC), and show the softening of distributions in the sum of energies of the first two hardest jets or in the $M_{C}$ variable. Since the majority of mass measurement techniques are based on edges in kinematic distributions, and these change appreciably when there is additional QCD radiation in the final state, the knowledge of higher-order QCD effects is required for precise mass determinations.
\end{abstract}

DOI: $10.1103 /$ PhysRevD.101.115012

\section{INTRODUCTION}

The discovery of dark matter (DM) in a collider experiment crucially depends on the ability to measure precisely its properties-its mass and couplings to visible matter. These are the necessary ingredients to test the hypothesis of a "WIMP" miracle [1-4]. Given the importance of such a discovery a number of methods to measure DM mass have been developed [5-18]. In this paper we are interested in understanding how QCD radiations modifies the precise determination of DM mass. Many of the methods for DM mass measurements were developed with low energy supersymmetry (SUSY) in mind [19]. We will thus also use SUSY as an example, though our results do apply more generally.

A significant effort was devoted in measuring DM mass at hadronic colliders. An ingenious method was put forward in [10,11], where it was applied to $\tilde{g} \rightarrow q \bar{q} \chi$ decays in gluino pair production. The mass of $\tilde{g}$ and $\chi$ can both be measured simultaneously from $m_{T 2}$, by computing for each event the value of $m_{T 2}$ as a function of an assumed $\chi$ mass, $m_{T 2}\left(m_{\text {trial }}\right)$. The envelope of

Published by the American Physical Society under the terms of the Creative Commons Attribution 4.0 International license. Further distribution of this work must maintain attribution to the author(s) and the published article's title, journal citation, and DOI. Funded by SCOAP ${ }^{3}$.
$m_{T 2}\left(m_{\text {trial }}\right)$ curves exhibits a kink at $m_{\text {trial }}=m_{\chi}$, where $m_{T 2}=m_{\tilde{g}}$. Measuring the kink determines both masses (for the effect of radiative corrections see [20]). For two body decays, e.g., for squark decays, $\tilde{q} \rightarrow q \chi$, the kinks in the distributions appear only once initial state radiation is included [7]. This underscores the importance of radiative corrections for DM mass measurement using kinematical distributions.

In this paper we explore a somewhat simpler case- the squark pair production in $e^{+} e^{-}$collisions. We focus on a two body decay, $\tilde{q} \rightarrow q \chi$, with $q$ a light quark and $\chi$ a neutralino (for earlier work see [21-23]). Emission of a hard gluon converts this to a three body decay, $\tilde{q} \rightarrow q g \chi$, qualitatively changing the kinematical distributions. Hard gluon emissions, on the other hand, are relatively rare, suppressed by small coupling constant, $\alpha_{s} \lesssim 0.09$ for $m_{\tilde{q}} \gtrsim 1 \mathrm{TeV}$. Most commonly the radiated gluons are either soft or collinear with the outgoing quark, affecting the kinematical distributions in the endpoint region where the decay is almost twobody. Parametrizing the neutralino energy in the squark rest frame as

$$
E_{\chi}=\frac{z M}{2}+\frac{m_{\chi}^{2}}{2 z M}
$$

the endpoint region is given by $z \sim 1$. Here $M\left(m_{\chi}\right)$ is the squark (neutralino) mass, while the dimensionless variable $z$ 
takes values, $z \in\left[m_{\chi} / M, 1\right]$. Near the end-point the neutralino is maximally boosted and $z$ becomes close to 1 .

The collinear and soft singularities of QCD contributions in the endpoint regions lead to large logarithms, $L \sim \ln (1-z)$, in the calculation of the differential decay width, $d \Gamma / d z$. Working to next-to-leading order (NLO) in $\alpha_{s}$, i.e., to $\mathcal{O}\left(\alpha_{s}\right)$, the Sudakov effects result in large double logarithmic contributions of the form $\alpha_{s} L^{2}$. In order to obtain reliable predictions, these logarithms need to be resummed to all orders in $\alpha_{s}$. At next-to-next-to-leading logarithmic (NNLL) accuracy the resummed decay width is given by

$$
\ln \frac{d \Gamma}{d z}=L f_{0}\left(\alpha_{s} L\right)+f_{1}\left(\alpha_{s} L\right)+\alpha_{s} f_{2}\left(\alpha_{s} L\right),
$$

with $f_{i}(\ldots)$ dimensionless functions that are $\mathcal{O}(1)$, counting the large logarithms as $L \sim 1 / \alpha_{s}$. This shows explicitly the dominance of the endpoint region, where the first term on the right-hand side (rhs) is the leading contribution. Keeping just the first term would give the result for decay width at leading logarithmic (LL) accuracy, obtained by resumming the double logarithms in the perturbative expansion of the form $\exp \left(L f_{0}\left(\alpha_{s} L\right)\right)=\sum_{k=0} a_{k}\left(\alpha_{s} L^{2}\right)^{k}$. The second and the third terms on the rhs in Eq. (2), of NLL and NNLL accuracy, then resum terms that are additionally suppressed by $\alpha_{s}$ and $\alpha_{s}^{2}$, respectively.

To resum the endpoint logarithms we employ softcollinear effective theory (SCET) [24-26], which properly describes collinear and soft gluon radiation in the endpoint region. The squark decay near the endpoint is governed by three distinct scales: hard $\left(\mu_{H}\right)$, jet $\left(\mu_{J}\right)$, and soft $\left(\mu_{S}\right)$ scales. For large mass splittings, $M-m_{\chi} \sim O(M)$, the hard scale $\mu_{H}$ can be identified with $\mu_{H} \sim M$. The light quark together with radiated collinear gluons forms a collimated jet, controlled by a typical scale $\mu_{J} \sim M \sqrt{1-z}$. Finally, the soft gluon radiations arise at the scale $\mu_{S} \sim M(1-z)$. Note that the kinematics of this problem is very similar to the decay $B \rightarrow X_{s} \gamma$ in the endpoint region, i.e., in the part of the phase space where the final state photon is close to maximally boosted. The effects of strong interactions are in this case described by collinear and soft gluon radiations. The factorization formalism for $B \rightarrow X_{s} \gamma$ near the endpoint was established in Refs. [24,26,27].

Similarly to $B \rightarrow X_{s} \gamma$, the differential decay width for $\tilde{q} \rightarrow q \chi$ can be schematically factorized as

$$
\frac{d \Gamma}{d z}=H\left(M, \mu_{F}\right) J\left(M \sqrt{1-z}, \mu_{F}\right) \otimes S\left(M(1-z), \mu_{F}\right),
$$

where $H, J$, and $S$ are the hard, jet, and collinear functions, respectively. The $\otimes$ denotes the appropriate convolution over $1-z$, while $\mu_{F}$ is the factorization scale. The decay width is independent of the factorization scale, which means that $\mu_{F}$ can be chosen arbitrarily. In general there will be large hierarchies between $\mu_{F}$ and $\mu_{H, J, S}$, so that one needs to perform renormalization group (RG) evolution for each of the $H, J$ and $S$ functions. These RG evolutions in SCET automatically resum the large endpoint logarithms.

Away from the endpoint region, where $1-z \sim O(1)$, the differential rate is dominated by hard gluon emissions from squark and quark lines, giving the event rate that is $\mathcal{O}\left(\alpha_{s}\right)$. This is of the same order as the NNLL corrections in the endpoint region and thus needs to be kept in our expressions. We compute these contributions using fixed order calculation at NLO in $\alpha_{s}$. We smoothly connect the two expressions, valid in the endpoint region and away from the endpoint regions, giving our final result for the decay width distribution at NNLL + NLO accuracy. We use the obtained expressions to perform a numerical study of the impact of QCD corrections in $e^{+} e^{-} \rightarrow \tilde{q} \tilde{q}^{*}$ events, using a weighted Monte-Carlo simulation.

To compare directly with the experiment our results for the decay widths will still need to be supplemented with a resummation of soft and Coulomb gluon radiation contributions connecting the two squarks, see Refs. [28,29] for LHC. These are especially important for slowly moving squarks, i.e., at threshold productions, and can even lead to squark bound states [28,30-32].

The paper is structured as follows. In Sec. II, we introduce the necessary ingredients of the effective field theory (EFT) approach to the problem, that includes SCET and heavy scalar effective theory (HSET). The HSET describes soft fluctuations of the heavy squark arising from soft gluon radiations. The HSET and SCET are then used to derive the factorization theorem for the squark decay rate near endpoint in Sec. III. The NLO calculation of the decay width in the full kinematical range of $z$ is obtained in Sec. IV. Using our results that combine the resummed and fixed calculations, giving the NNLL + NLO accuracy, we perform in Sec. V a phenomenological study of squark pair production in $e^{+} e^{-}$annihilation, and then conclude in Sec. VI. Appendix A contains technical details on $\Delta$-distribution which has been used to regularize infrared (IR) divergences in the fixed NLO calculation.

\section{CONSTRUCTION OF EFFECTIVE THEORY LAGRANGIAN}

We are interested in the squark decay, $\tilde{q} \rightarrow q \chi$, where $\chi$ is the dark matter (DM) particle, and how this is affected by QCD radiation. Near the endpoint, $\chi$ and a collimated jet are almost back-to-back in the squark rest frame. DM, $\chi$, escapes detection and manifests itself in the detector as missing energy. The quark interacts strongly-it radiates collinear gluons and quark-antiquark pairs, which form a collimated jet. In addition, there is soft gluon radiation in the event, which does not have a preferred direction.

As explained in the Introduction, the decay is governed by three distinct scales, $\mu_{H}, \mu_{J}$, and $\mu_{S}$. We use EFTs to deal with the hierarchies between the three scales and the 
associated large logarithms. We first integrate out the hard interactions, where the relevant hard scale, $\mu_{H}$, is comparable to the squark mass $M$. At energy scales below $\mu_{H}$ we then have only collinear and soft degrees of freedom. The light quark and the collinear gluon describe collinear interactions for the collimated jet. Also the soft mode decoupled from the collinear quark and the heavy squark describes soft gluon radiations near the endpoint. SCET is the appropriate EFT that describes collinear and soft modes and their interactions. It provides a systematic way to decouple soft modes from the collinear field. This is very useful when proving factorization in the endpoint region. The interactions of heavy squark are described by the HSET, which is obtained by integrating out the hard gluon modes and the squark mass $M$. In the rest of this section we show how SCET and HSET are constructed. The decay rate of the heavy squark is calculated in the subsequent section.

\section{A. Decay Lagrangian at the hard scale}

We take $\chi$ to be a Majorana fermion. This is the case in the MSSM where $\chi$ is the lightest supersymmetric particle (LSP)_-assumed to be the lightest neutralino. The most general Lagrangian describing a two-body decay of a color triplet scalar, $\tilde{q}$, to a quark, $q$, and a Majorana fermion $\chi$, is given by ${ }^{1}$

$$
\begin{aligned}
\mathcal{L}_{\text {int }} & =\sum_{L, R} B_{i}(\mu) O_{i}(\mu)+\text { H.c. } \\
& =B_{L}(\mu)\left(\bar{q}_{L} P_{R} \chi\right) \tilde{q}+B_{R}(\mu)\left(\bar{q}_{R} P_{L} \chi\right) \tilde{q}+\text { H.c. },
\end{aligned}
$$

where we are using the four-component notation with $P_{L, R}=\left(1 \mp \gamma_{5}\right) / 2$. The dimensionless Wilson coefficients $B_{L, R}$ encode the new physics as well as strong interactions above the hard scale $\mu_{H} \sim M$. Our analysis applies to MSSM, but is also more general and applies to any decays of the form $\tilde{q} \rightarrow q \chi$, where $\tilde{q}$ is a color triplet scalar.

In the MSSM for each quark flavor there are two squarks, $\tilde{q}_{1,2}$, so that the above Lagrangian modifies to

$$
\mathcal{L}_{\text {int }}=\sum_{i=1,2} B_{L i}(\mu)\left(\bar{q}_{L} P_{R} \chi\right) \tilde{q}_{i}+B_{R i}(\mu)\left(\bar{q}_{R} P_{L} \chi\right) \tilde{q}_{i}+\text { H.c. }
$$

The tree level expressions for the Wilson coefficients are, neglecting flavor violating effects,

$$
B_{L i}=C_{L L} L_{\tilde{q}_{i}}+C_{L R} R_{\tilde{q}_{i}}, \quad B_{R i}=C_{R L} L_{\tilde{q}_{i}}+C_{R R} R_{\tilde{q}_{i}},
$$

with

\footnotetext{
${ }^{1}$ For a Dirac fermion $\chi$ there are two additional terms in (4), $B_{L}^{\prime}(\mu)\left(\bar{q}_{L} P_{R} \chi^{c}\right) \tilde{q}$ and $B_{R}^{\prime}(\mu)\left(\bar{q}_{R} P_{L} \chi^{c}\right) \tilde{q}$.
}

$$
\begin{aligned}
& C_{L L}=-\sqrt{2}\left[g T_{3}^{q} N_{12}+g^{\prime}\left(Q_{q}-T_{3}^{q}\right) N_{11}\right], \\
& C_{R R}=\sqrt{2} g^{\prime} Q_{q} N_{11}^{*}, \\
& C_{R L}=C_{L R}^{*}=-\sqrt{2} m_{q}\left(N_{14}^{*} \delta_{q u}+N_{13}^{*} \delta_{q d}\right) / v,
\end{aligned}
$$

with $g, g^{\prime}$ the weak and hypercharge gauge couplings, $Q_{q}$ the electric charge of quark $q$, and $T_{3}^{q}$ the weak isospin, while $R_{\tilde{q}_{1}}=L_{\tilde{q}_{2}}^{*}=\cos \left(\theta_{\tilde{q}}\right)$, and $L_{\tilde{q}_{1}}=-R_{\tilde{q}_{2}}^{*}=\sin \left(\theta_{\tilde{q}}\right)$, with $\theta_{\tilde{q}}$ the mixing angle rotating the squark gauge eigenstates $\tilde{q}_{R, L}$ to mass eigenstates $\tilde{q}_{1,2}$. The $\tilde{q}_{R}-\tilde{q}_{L}$ mixing is usually important only for the third generation squarks, while for the first two generations gauge and mass eigenstates coincide, $\theta_{\tilde{q}}=0$. The neutralino mixing matrix is denoted by $N_{i j}$. If LSP is mostly gaugino then $N_{11,12} \gg$ $N_{13,14}$ and thus $\tilde{q}_{L} \rightarrow q_{L} \chi$ and $\tilde{q}_{R} \rightarrow q_{R} \chi$ for the first two generations. For well-tempered neutralino, on the other hand, all terms in (7), (8) may be important.

\section{B. EFTs for the endpoint region}

We restrict ourselves to the case where quark mass can be neglected compared to $M$. We will work in the squark rest frame, so that its four-velocity $v^{\mu}$ is given by $v^{\mu}=(1, \mathbf{0})$. We orient the coordinate system such that jet goes in the $z$ direction, i.e., that, neglecting its mass, it is on the light cone $n^{\mu}=(1,0,0,1)$. We also introduce the opposite light cone four-vector $\bar{n}^{\mu}=(1,0,0,-1)$, so that $n^{2}=\bar{n}^{2}=0$, $n \cdot \bar{n}=2$ and $p_{\tilde{q}}^{\mu}=M v^{\mu}=M\left(n^{\mu}+\bar{n}^{\mu}\right) / 2$. We will use light-cone coordinates, in which a four-momentum $p^{\mu}$ is given by $p^{\mu}=\left(\bar{n} \cdot p, p_{\perp}, n \cdot p\right)$.

The effective field theory to reproduce low energy physics in full QCD is obtained by integrating hard degrees of freedom. For instance, the hard gluon exchanges between the heavy squark and the light quark are integrated out. The Wilson coefficients $B_{L, R}$ in Eq. (4) thus get modified to $\mathcal{C}_{L, R}(\mu)$ (see Eq. (13) below). The resultant EFT is valid at the scale $\mu<\mu_{H} \sim M$. And the remaining degrees of freedom in EFT are collinear and soft fields scaling as $p_{c}=M\left(1, \lambda, \lambda^{2}\right)$ and $p_{s}=M\left(\lambda^{2}, \lambda^{2}, \lambda^{2}\right)$ respectively. Here $\lambda$ is a small expansion parameter in EFT. For the squark decay near endpoint, $\lambda$ is given as $\sim \sqrt{1-z}$.

In the heavy squark sector, after integrating out hard fluctuations as well as heavy squark mass $M$, the heavy squark only interacts with soft gluons. Then full QCD Lagrangian for the heavy squark can be matched onto HSET Lagrangian,

$$
\mathcal{L}_{\mathrm{HSET}}=\phi_{v}^{*} v \cdot i D_{s} \phi_{v}-\frac{1}{2 M} \phi_{v}^{*} D_{s}^{2} \phi_{v}+\mathcal{O}\left(1 / M^{2}\right),
$$

where $\phi_{v}$ is the squark field in HSET,

$$
\tilde{q}(x)=\frac{1}{\sqrt{2 M}} e^{-i M v \cdot x} \phi_{v}(x),
$$


The covariant derivative $D_{s}^{\mu}=\partial^{\mu}-i g A_{s}^{\mu, a} T^{a}$ includes only the soft gluon field. The second term in (9) is $\mathcal{O}(1 / M)$ with a coefficient that is fixed by reparametrization invariance. We work at leading order in $1 / M$ expansion, and thus only keep the first term in (9).

The light quark field matches onto $n$-collinear field in SCET so that

$$
q(x)=\sum_{\tilde{p}} e^{-i \tilde{p} \cdot x} q_{n, p}(x)=\sum_{\tilde{p}} e^{-i \tilde{p} \cdot x}\left(\xi_{n, p}(x)+\xi_{\bar{n}, p}(x)\right),
$$

where

$$
\xi_{n, p}(x)=\frac{n \bar{n}}{4} q_{n, p}(x), \quad \xi_{\bar{n}, p}(x)=\frac{\bar{n} n}{4} q_{n, p}(x),
$$

and thus $n \xi_{n, p}=\bar{n} \xi_{\bar{n}, p}=0$. The summation is over large label momenta given by $\tilde{p}^{\mu}=\bar{n} \cdot p n^{\mu} / 2+p_{\perp}^{\mu}$ that differ by soft fluctuations. The field $\xi_{\bar{n}}$ is suppressed by $\lambda$, and is thus not present as an external quark field in our analysis of squark decays since we work to leading order (LO) in the $1 / M$ expansion. Integrating out $\xi_{\bar{n}}$ the collinear interactions can be expressed entirely in terms of $\xi_{n}$. The resulting LO SCET Lagrangian for collinear fields can be found in, e.g., Ref. [25].

The decay Lagrangian (4) matches onto the HSET + SCET effective decay Lagrangian, appropriate for describing the squark decays in the endpoint region,

$$
\begin{aligned}
\mathcal{L}_{\text {int }}^{\text {eff }}= & {\left[\sum_{\tilde{p}} \frac{\mathcal{C}_{L}(\mu)}{\sqrt{2 M}} e^{-i(M v-\tilde{p}) \cdot x}\left(\bar{\xi}_{n, p} W_{n} P_{R} \chi\right) \phi_{v}(x)+\text { H.c. }\right] } \\
& +[L \leftrightarrow R] \\
\equiv & \sum_{\tilde{p}}\left[e^{-i(M v-\tilde{p})} \mathcal{C}_{L}(\mu) \mathcal{O}_{L}(x, \mu)+\text { H.c. }\right] \\
& +\sum_{\tilde{p}}\left[e^{-i(M v-\tilde{p})} \mathcal{C}_{R}(\mu) \mathcal{O}_{R}(x, \mu)+\text { H.c. }\right],
\end{aligned}
$$

In the sum only the $\tilde{p}$ that satisfy momentum conservation are selected. The hard gluon exchanges are encoded in Wilson coefficients $\mathcal{C}_{L, R}$ [obtained from $B_{L, R}$ in (4)], while collinear gluons emitted from the heavy squark yield the collinear Wilson line

$$
W_{n}(x)=\mathrm{P} \exp \left(i g \int_{-\infty}^{x} d s \bar{n} \cdot A_{n}^{a}\left(s \bar{n}^{\mu}\right) T^{a}\right) .
$$

Here $A_{n}^{\mu}$ is $n$-collinear gluon field and "P" indicates the path-ordered integral.

To show the factorization of soft and collinear interactions it is useful to perform field redefinitions, $\xi_{n} \rightarrow Y_{n} \xi_{n}, A_{n}^{\mu} \rightarrow Y_{n} A_{n}^{\mu} Y_{n}^{\dagger}$, and $\phi_{v} \rightarrow Y_{v} \phi_{v}$ [26], factoring out the soft Wilson lines in the $n$ and $v$ directions, $Y_{n, v}$,
$Y_{\mathrm{v}}(x)=\operatorname{Pexp}\left(i g \int_{-\infty}^{x} d s \mathrm{v} \cdot A_{s}^{a}\left(s \mathrm{v}^{\mu}\right) T^{a}\right), \quad \mathrm{v}^{\mu}=n^{\mu}, v^{\mu}$.

The path of integration over $s \in[-\infty, x]$ indicates that the dressed collinear or squark field is incoming. For the outgoing particles the integration path is over $s \in[x,+\infty]$, giving for the soft Wilson lines [33],

$\tilde{Y}_{\mathrm{v}}^{\dagger}(x)=\operatorname{Pexp}\left(i g \int_{x}^{+\infty} d s \mathrm{v} \cdot A_{s}^{a}\left(s \mathrm{v}^{\mu}\right) T^{a}\right), \quad \mathrm{v}^{\mu}=n^{\mu}, v^{\mu}$.

In the LO SCET and HSET Lagrangian the interactions between soft gluons and the redefined collinear fields, $\xi_{n}, A_{n}^{\mu}$, and between the soft gluons and the heavy squark field $\phi_{v}$, drop out (that is, at LO there are no interactions between collinear and soft fields, and no interactions between redefined heavy squark and soft fields). The effects of soft gluons are thus moved into the effective decay Lagrangian, where they appear as a product of two soft Wilson lines in $n$ and $v$ directions,

$$
\mathcal{L}_{\text {int }}^{\text {eff }}=\sum_{i=L, R} \sum_{\tilde{p}} \mathcal{C}_{i}(\mu) e^{-i(M v-\tilde{p}) \cdot x} \mathcal{O}_{i}^{a} \chi_{a}+\text { H.c. },
$$

with

$$
\mathcal{O}_{L, R}^{a}(\mu)=\frac{1}{\sqrt{2 M}}\left(\bar{\xi}_{n, p} W_{n} P_{R, L}\right)^{a} \tilde{Y}_{n}^{\dagger} Y_{v} \phi_{v}(x) .
$$

In Eq. (17) a summation over Dirac four-component index $a$ is implied. From now on we will use the form of EFT Lagrangian given in Eq. (17), i.e., with $\xi_{n}, A_{n}^{\mu}$, and $\phi_{v}$ denoting the redefined fields that do not couple to soft gluons and quarks at $\mathrm{LO}$.

\section{DIFFERENTIAL DECAY RATE AT THE ENDPOINT}

The total decay rate for $\tilde{q} \rightarrow \chi^{0} q_{L}$ averaged over the squark color is

$\Gamma\left(\tilde{q} \rightarrow \chi^{0} q_{L}\right)=\frac{1}{2 M} \int \frac{d^{3} p_{\chi}}{(2 \pi)^{3}} \frac{1}{2 E_{\chi}} \mathcal{T}_{L}\left(E_{\chi}, m_{\chi}, M\right)$,

where $\mathcal{T}_{L}\left(E_{\chi}, m_{\chi}, M\right)$ is related to the matrix elements squared for squark decays into left-handed quarks

$\mathcal{T}_{L}\left(E_{\chi}, m_{\chi}, M\right)=\sum_{X}(2 \pi)^{4} \delta\left(p-p_{\chi}-p_{X}\right)\left|\mathcal{M}_{L}\right|^{2}$

Explicitly, the matrix elements squared are 


$$
\begin{aligned}
\left|\mathcal{M}_{L}\right|^{2}= & \left|B_{L}(\mu)\right|^{2}\left\langle\tilde{q}\left|\tilde{q}^{* \alpha}\left(P_{L} q^{\alpha}\right)_{a}\right| X\right\rangle \\
& \times\left\langle X\left|\left(\bar{q}^{\beta} P_{R}\right)_{b} \tilde{q}^{\beta}\right| \tilde{q}\right\rangle\left(p_{\chi}-m_{\chi}\right)_{b a},
\end{aligned}
$$

with the summation over color indices, $\alpha, \beta$, and Lorentz indices, $a, b$ implied. We do not show color index of squark external state: we always consider color-averaged initial states, hence $\langle\tilde{q}|\cdots| \tilde{q}\rangle=\left\langle\tilde{q}^{\alpha}|\cdots| \tilde{q}^{\alpha}\right\rangle / N_{c}$ in our convention. Note that in Eq. (21) we already used the fact that neutralinos are not charged under QCD and thus only contribute as $\sum_{\text {spin }} u_{b} \bar{u}_{a}=\left(p_{\chi}-m_{\chi}\right)_{b a}$. For $\tilde{q} \rightarrow \chi^{0} q_{R}$ decays the same results apply, but with $L \leftrightarrow R$. The interference terms between the two decays are $m_{q} / M$ suppressed and can be safely neglected.

At tree level the decay rate is given by

$$
\Gamma^{(0)}\left(\tilde{q} \rightarrow \chi^{0} q_{L}\right)=\frac{\left|B_{L}\right|^{2}}{16 \pi} M\left(1-r_{\chi}\right)^{2}
$$

with

$$
r_{\chi}=m_{\chi}^{2} / M^{2}
$$

\section{A. Factorization theorem near the endpoint}

We start by reviewing the decay kinematics near the endpoint, where in the final states we have an energetic neutralino and a collimated jet as well as soft gluons. Following Eq. (1), we define the kinematic variable

$$
z=\frac{n \cdot p_{\chi}}{M}
$$

in terms of which the neutralino and other final states momenta are given by

$$
\begin{aligned}
& p_{\chi}^{\mu}=\frac{M}{2}\left(\frac{r_{\chi}}{z} n^{\mu}+z \bar{n}^{\mu}\right), \\
& p_{X} \equiv p_{J}^{\mu}+p_{S}^{\mu}=\frac{M}{2}\left[\left(1-\frac{r_{\chi}}{z}\right) n^{\mu}+(1-z) \bar{n}^{\mu}\right] .
\end{aligned}
$$

Here $z$ can take values $z \in\left[\sqrt{r_{\chi}}, 1\right]$ (we oriented the coordinate system such that $\vec{p}_{\chi}$ is always along negative $z$-axis, while $\vec{p}_{X}$ is along positive $z$-axis). The missing energy is $E_{\chi}=v \cdot p_{\chi}=\left(r_{\chi} / z+z\right) M / 2$, and therefore one can use $z$ and $E_{\chi}$ interchangeably. The invariant mass of the collinear and soft final states is $p_{X}^{2}=M^{2}\left(1-r_{\chi} / z\right)(1-z)$. The limit of a very collimated jet, $p_{X}^{2} \rightarrow 0$, is thus obtained in the limit $z \rightarrow 1$.

In the endpoint region, $1-z \ll 1$, we can apply the HSET and SCET formalism, introduced in the previous section. The differential rate is

$$
\frac{d \Gamma\left(\tilde{q} \rightarrow \chi^{0} q_{L}\right)}{d E_{\chi}}=\frac{\left(E_{\chi}^{2}-p_{\chi}^{2}\right)^{1 / 2}}{8 \pi^{2} M} \mathcal{T}_{L}\left(E_{\chi}, m_{\chi}, M\right),
$$

with $\mathcal{T}_{L}=\sum_{X}(2 \pi)^{4} \delta\left(p-p_{\chi}-p_{X}\right)\left|\mathcal{M}_{L}\right|^{2}$, where $\left|\mathcal{M}_{L}\right|^{2}$ is calculated in HSET + SCET,

$$
\begin{aligned}
\left|\mathcal{M}_{L}\right|^{2}= & 2 M\left|\mathcal{C}_{L}(\mu)\right|^{2}\left\langle\phi_{v}\left|\mathcal{O}_{L, a}^{\dagger}\right| X\right\rangle \\
& \times\left\langle X\left|\mathcal{O}_{L, b}\right| \phi_{v}\right\rangle\left(p_{\chi}-m_{\chi}\right)_{b a}+\mathcal{O}(1 / M) .
\end{aligned}
$$

The EFT operators $\mathcal{O}_{L, R}$ are given in Eq. (18), and $|\tilde{q}\rangle=$ $\sqrt{2 M}\left|\phi_{v}\right\rangle$ up to $1 / M$ corrections. The Wilson coefficients $\mathcal{C}_{L, R}$ can be decomposed into

$$
\mathcal{C}_{L, R}=B_{L, R} \times C_{L, R},
$$

where $B_{L, R}$ are the unknown new physics Wilson coefficients in Eq. (4) and $C_{L, R}$ are the Wilson coefficients as a result of integrating out the squark mass $M$ and the hard gluon exchanges between squark and quark. The NLO matching onto HSET + SCET has been performed in Sec. III B, with the result for $C_{L, R}$ given in Eq. (37).

In the remainder of this section we use the operator production expansion (OPE) to arrive at a more practically useful expression for $\mathcal{T}_{L}$. We first use the completeness relation, $\sum_{X}|X\rangle\langle X|=1$, to rewrite $\mathcal{T}_{L}$ as (neglecting $\mathcal{O}(1 / M)$ corrections $)$

$$
\begin{aligned}
\mathcal{T}_{L}= & 2 M\left|\mathcal{C}_{L}\left(M, m_{\chi}, \mu\right)\right|^{2} \\
& \times \int d^{4} y e^{i\left(M v-p_{\chi}-\tilde{p}_{c}\right) \cdot y}\left(p_{\chi}-m_{\chi}\right)_{b a} \\
& \times\left\langle\phi_{v}\left|\mathcal{O}_{L, a}^{\dagger}(y) \mathcal{O}_{L, b}(0)\right| \phi_{v}\right\rangle .
\end{aligned}
$$

The large label momentum $\tilde{p}_{c}$ is the $n^{\mu}$-component of $p_{X}^{\mu}$, given in Eq. (25). The phase in (29) is therefore equal to

$$
M v^{\mu}-p_{\chi}^{\mu}-\tilde{p}_{c}^{\mu}=M(1-z) \bar{n}^{\mu} / 2,
$$

so that

$$
\begin{aligned}
& \mathcal{T}_{L}\left(E_{\chi}, m_{\chi}, M\right) \\
&= z M^{2}\left|\mathcal{C}_{L}\left(M, m_{\chi}, \mu\right)\right|^{2} \int d^{4} y e^{i M(1-z) y_{-} / 2} \\
& \times\langle 0|\left(\tilde{Y}_{n}^{\dagger} Y_{v}\right)^{\alpha \beta}(y)\left(W_{n}^{\dagger} P_{L} \xi_{n}(y)\right)_{a}^{\beta} \\
& \times\left(\bar{\xi}_{n} P_{R} \bar{n} W_{n}\right)_{a}^{\gamma}(0)\left(\tilde{Y}_{n} Y_{v}^{\dagger}\right)^{\gamma \alpha}(0)|0\rangle,
\end{aligned}
$$

where we used the shorthand notation $y_{-}=\bar{n} \cdot y$ $\left(y_{+}=n \cdot y\right)$ and that $\phi_{v}^{\alpha}\left|\phi_{v}^{\beta}\right\rangle=\delta^{\alpha \beta}$.

The collinear field $\xi_{n}$ describes an inclusive jet in $n$-direction. The corresponding jet function is defined as 


$$
\begin{aligned}
& \left\langle 0\left|\left(W_{n}^{\dagger} \xi_{n, p}(y)\right)_{a}^{\alpha}\left(\bar{\xi}_{n} W_{n, p}\right)_{b}^{\beta}(0)\right| 0\right\rangle \\
& =\delta^{\alpha \beta}\left(\frac{n}{2}\right)_{a b} \int \frac{d^{4} k}{(2 \pi)^{3}} e^{-i k \cdot y} J_{n}\left(k_{+} ; \bar{n} \cdot \tilde{p}\right) \\
& =\delta^{\alpha \beta}\left(\frac{n}{2}\right)_{a b} \delta\left(y_{+}\right) \delta\left(y_{\perp}\right) \int d k_{+} e^{-i k_{+} y_{-} / 2} J_{n}\left(k_{+} ; \bar{n} \cdot \tilde{p}\right) .
\end{aligned}
$$

At LO in $\alpha_{s}$ the jet function is simply $J_{n}^{(0)}=\delta\left(k_{+}\right)$.

The product of Wilson lines in Eq. (31) forms the soft function $S\left(l_{+}\right)$, defined as

$$
S\left(l_{+}, \mu\right)=\operatorname{Tr}\left\langle 0\left|Y_{v}^{\dagger} \tilde{Y}_{n} \delta\left(l_{+}+n \cdot i \partial\right) \tilde{Y}_{n}^{\dagger} Y_{v}\right| 0\right\rangle,
$$

so that its Fourier transform is

$$
\operatorname{Tr}\left\langle 0\left|Y_{v}^{\dagger} \tilde{Y}_{n}\left(y_{-}\right) \tilde{Y}_{n}^{\dagger} Y_{v}(0)\right| 0\right\rangle=\int d l_{+} e^{-i l_{+} y_{-} / 2} S\left(l_{+}\right) .
$$

The expression for $\mathcal{T}_{L}$ in Eq. (31) can therefore be written as the convolution of the soft function, Eq. (34), and the jet function, Eq. (32),

$$
\begin{aligned}
& \mathcal{T}_{L}\left(z, m_{\chi}, M\right) \\
& =4 \pi\left|\mathcal{C}_{L}\left(M, m_{\chi}, \mu\right)\right|^{2} z M^{2} \\
& \quad \times \int d l_{+} S\left(l_{+}, \mu\right) J_{n}\left(M(1-z)-l_{+}, \mu ; M\left(1-r_{\chi} / z\right)\right) .
\end{aligned}
$$

Collecting all the terms, the differential decay rate $d \Gamma / d z$ in the $z \rightarrow 1$ limit can thus be written as

$$
\begin{aligned}
\frac{d \Gamma}{d z}(\tilde{q} & \left.\rightarrow \chi^{0} X_{q_{L}}\right) \\
= & \frac{M^{2}}{16 \pi}\left|\mathcal{C}_{L}(M, x, \mu)\right|^{2}\left(z-\frac{r_{\chi}}{z}\right)^{2} \\
& \times \int d l_{+} J_{n}\left(M(1-z)-l_{+}, \mu ; M\left(1-r_{\chi} / z\right)\right) S\left(l_{+}, \mu\right),
\end{aligned}
$$

where the large momentum, $M\left(1-r_{\chi} / z\right)$, in the jet function can be further simplified to $M\left(1-r_{\chi}\right)$, neglecting $\mathcal{O}(1-z)$ corrections.

The factorization formula (36) is similar to the one for $B \rightarrow X_{s} \gamma \quad[24,26,27]$. The main difference are the hard interactions. Another difference is that the soft function in the $\tilde{q} \rightarrow \chi q$ decay would be treated perturbatively. For the squark mass $M \gtrsim O(1 \mathrm{TeV})$, the typical soft scale $\mu_{S} \sim$ $M(1-z)$ would be of a few tens of $\mathrm{GeV}$, and it is much larger than the hadronic scale $\Lambda_{\mathrm{QCD}} \lesssim 1 \mathrm{GeV}$. Unlike $B \rightarrow X_{s} \gamma$, where the predictions in the endpoint region are given in terms of the nonperturbative $B$ meson shape functions, the nonperturbative physics here affects the region of phase space less than $1-z \sim \mathcal{O}\left(\Lambda_{\mathrm{QCD}} / M\right) \sim 10^{-3}$, which does not significantly change our phenomenological conclusions in Sec. V B.

\section{B. Radiative corrections}

We now return to the calculation of $C_{L, R}$ in Eq. (28). At scale $\mu \sim M$, we need to integrate out the heavy squark mass $M$ and the hard gluon fluctuations of order $M$, matching onto HSET + SCET. For this we match QCD calculations in $\alpha_{s}$ for the effective operators in full QCD [Eq. (4)] and ones in SCET + HSET [Eq. (13)]. And we obtain the Wilson coefficients $C_{L, R}$ at the higher order in $\alpha_{s}$. At tree level the matching is trivial, resulting in $C_{L, R}=1$.

For NLO results of $C_{L, R}$ we compute Feynman diagrams shown in Fig. 1 and self energy diagrams at one loop.

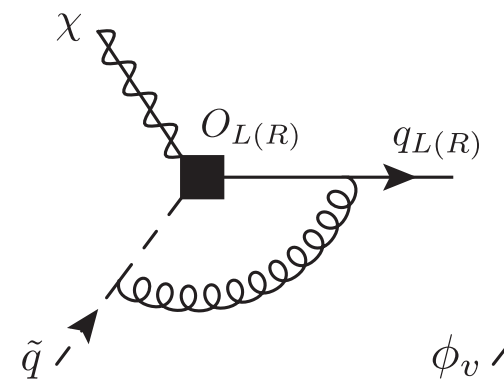

(a)

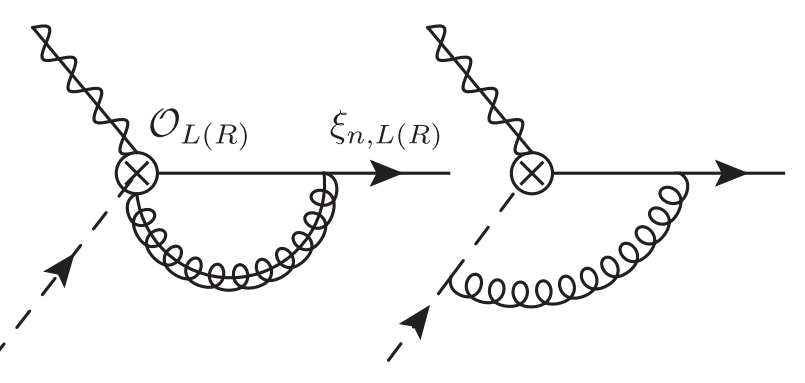

(b)

FIG. 1. One loop diagrams for matching between full QCD (a) and SCET + HSET (b). Here $O_{L(R)}=\left(\bar{q}_{L(R)} P_{R(L)} \chi\right) \tilde{q}$ and $\mathcal{O}_{L(R)}=\left(\bar{\xi}_{n, L(R)} W_{n} P_{R(L)} \chi\right) \phi_{v}$. In diagrams (b), the first (second) diagram is for collinear (soft) gluon exchange. Self-energy diagrams are also needed for the matching process. 
Throughout this paper, we employ dimensional regularization in the $\overline{\mathrm{MS}}$ scheme with $D=4-2 \epsilon$ in order to handle ultraviolet (UV) singularity. As a result we obtain

$$
\begin{aligned}
C_{L, R}\left(M, r_{\chi}, \mu\right)= & 1+\frac{\alpha_{s}}{4 \pi} C_{F}\left[-\left(\frac{9}{4}+\frac{\pi^{2}}{12}+\ln \frac{\mu^{2}}{M^{2}}+\frac{1}{2} \ln ^{2} \frac{\mu^{2}}{M^{2}}\right)\right. \\
& \left.+2 \ln \left(1-r_{\chi}\right)\left(1+\ln \frac{\mu^{2}}{M^{2}}\right)-\ln ^{2}\left(1-r_{\chi}\right)+2 L i_{2}\left(\frac{r_{\chi}}{r_{\chi}-1}\right)\right] .
\end{aligned}
$$

Here $L i_{2}(x)$ is the dilogarithm function. The anomalous dimensions for $C_{L, R}$ are given

$$
\gamma_{C}=\frac{\mu}{C_{L, R}(\mu)} \frac{d}{d \mu} C_{L, R}(\mu)=-\frac{\alpha_{s}}{2 \pi} C_{F}\left(1+\ln \frac{\mu^{2}}{M^{2}\left(1-r_{\chi}\right)^{2}}\right)+\mathcal{O}\left(\alpha_{s}^{2}\right)
$$

Note that full Wilson coefficients $\mathcal{C}_{L, R}=B_{L, R} C_{L, R}$ involve the unknown new physics Wilson coefficients $B_{L, R}$. But, since the effective interaction Lagrangian in (13) should be scale invariant, we can compute $\gamma_{\mathcal{C}_{L, R}}$ by considering the renormalization behavior of the effective operators $\mathcal{O}_{L, R}$ in SCET + HSET. The relation between the bare and renormalized effective operators can be written as $Z_{\mathcal{O}}^{L, R} \mathcal{O}_{L, R}^{R}=Z_{\phi_{v}}^{1 / 2} Z_{\xi}^{1 / 2} \mathcal{O}_{L, R}^{B}$. Here,

$Z_{\phi_{v}}=1+\alpha_{s} C_{F} /(2 \pi \epsilon), \quad$ and $\quad Z_{\xi}=1-\alpha_{s} C_{F} /(4 \pi \epsilon)$,

are the wave function renormalizations for the heavy squark and the collinear quark fields respectively. Computing the one loop diagrams in Fig. 1(b) gives

$Z_{\mathcal{O}}^{L}=Z_{\mathcal{O}}^{R}=1+\frac{\alpha_{s}}{4 \pi} C_{F}\left[\frac{1}{\epsilon^{2}}+\frac{1}{\epsilon}\left(\frac{5}{2}+\ln \frac{\mu^{2}}{(\bar{n} \cdot p)^{2}(n \cdot v)^{2}}\right)\right]$,

where $\bar{n} \cdot p$ is the large momentum component for the quark and equals $\bar{n} \cdot p=M\left(1-r_{\chi}\right)$ in the $z \rightarrow 1$ limit, while $n \cdot v=1$ in the squark rest frame. From Eq. (40) we obtain the anomalous dimension for $\mathcal{C}_{L, R}$ satisfying the RG equation, $d /(d \ln \mu) \mathcal{C}_{L, R}=\gamma_{\mathcal{C}} \mathcal{C}_{L, R}$, as follows
$\gamma_{\mathcal{C}}=\left(\mu \frac{\partial}{\partial \mu}+\frac{\partial}{\partial g}\right) \ln Z_{\mathcal{O}}^{L, R}=-\frac{\alpha_{s}}{2 \pi} C_{F}\left(\frac{5}{2}+\ln \frac{\mu^{2}}{M^{2}\left(1-r_{\chi}\right)^{2}}\right)$.

Since $\mathcal{C}_{L, R}=B_{L, R} C_{L, R}$, from Eqs. (38) and (41) the anomalous dimensions for the unknown $B_{L, R}$ are obtained as

$$
\gamma_{B}=\frac{\mu}{B_{L, R}(\mu)} \frac{d}{d \mu} B_{L, R}(\mu)=-3 \frac{\alpha_{s}}{4 \pi} C_{F}+\mathcal{O}\left(\alpha_{s}^{2}\right) .
$$

In order to employ the standard plus distribution for the radiative corrections in Eq. (36), it is convenient to introduce dimensionless jet and soft functions,

$\bar{J}_{n}\left(x, \mu ; M\left(1-r_{\chi}\right)\right)=y M J_{n}\left(M(1-z)-l_{+}, \mu ; M\left(1-r_{\chi}\right)\right)$

$$
\bar{S}(y, \mu)=M S\left(l_{+}, \mu\right),
$$

where $y$ is related to $l_{+}$through

$$
l_{+}=M(1-y), \quad \text { while } x=z / y .
$$

The two new variables are defined in the interval $z \leq x$, $y \leq 1$. The limit of soft momenta in the soft function corresponds to $y \rightarrow 1$. In terms of the dimensionless jet and soft functions, the differential decay rate in Eq. (36) can be rewritten as

$$
\left.\frac{d \Gamma}{d z}\left(\tilde{q} \rightarrow \chi^{0} X_{q_{L}}\right)=\frac{M}{16 \pi}\left(z-\frac{r_{\chi}}{z}\right)^{2}\left|\mathcal{C}_{L}\left(M, r_{\chi}, \mu\right)\right|^{2} \int_{z}^{1} \frac{d x}{x} \bar{J}_{n}\left(x, \mu ; M\left(1-r_{\chi}\right)\right)\right) \bar{S}(z / x, \mu) .
$$

We computed the jet and the soft functions at next-to-leading order (NLO) in $\alpha_{s}$, and the results read [27,34]

$$
\bar{J}_{n}(x, \mu)=\delta(1-x)+\frac{\alpha_{s}}{2 \pi} C_{F}\left\{\delta(1-x)\left[\frac{7}{2}-\frac{\pi^{2}}{2}+\frac{3}{2} \ln \frac{\mu^{2}}{Q^{2}}+\ln ^{2} \frac{\mu^{2}}{Q^{2}}\right]-\frac{1}{(1-x)_{+}}\left[2 \ln \frac{\mu^{2}}{Q^{2}}+\frac{3}{2}\right]+2\left(\frac{\ln (1-x)}{1-x}\right)_{+}\right\},
$$


$\bar{S}(y, \mu)=\delta(1-y)+\frac{\alpha_{s}}{2 \pi} C_{F}\left\{\delta(1-y)\left[-\frac{\pi^{2}}{12}+\ln \frac{\mu^{2}}{M^{2}}-\frac{1}{2} \ln ^{2} \frac{\mu^{2}}{M^{2}}\right]+\frac{2}{(1-y)_{+}}\left[-1+\ln \frac{\mu^{2}}{M^{2}}\right]-4\left(\frac{\ln (1-y)}{1-y}\right)_{+}\right\}$,

where $Q^{2}=M^{2}\left(1-r_{\chi}\right)$ and $(\ldots)_{+}$the standard plus distribution. Note that both of the above results are infrared finite. The logarithms are minimized at $\mu=Q(1-x)^{1 / 2}$ and $\mu=M(1-y)$ for the jet and soft functions, respectively.

We can check that the obtained differential decay rate does not depend on the scale choice, $\mu$, to the order we are working. Differentiation with respect to $d(\log \mu)$ gives,

$$
\begin{aligned}
\mu \frac{d}{d \mu} \frac{d \Gamma}{d z}= & \frac{M}{16 \pi}\left(z-\frac{r_{\chi}}{z}\right)^{2}\left|\mathcal{C}_{L}(\mu)\right|^{2}\left\{2 \operatorname{Re}\left[\gamma_{\mathcal{C}}\right] \int_{z}^{1} \frac{d x}{x} \bar{J}_{n}(x, \mu) \bar{S}(z / x, \mu)\right. \\
& \left.+\int_{z}^{1} \frac{d x}{x}\left[\left(\mu \frac{d}{d \mu} \bar{J}_{n}(x, \mu)\right) \bar{S}(z / x, \mu)+\bar{J}_{n}(x, \mu)\left(\mu \frac{d}{d \mu} \bar{S}(z / x, \mu)\right)\right]\right\}, \\
= & \frac{M}{16 \pi}\left(z-\frac{r_{\chi}}{z}\right)^{2}\left|\mathcal{C}_{L}(\mu)\right|^{2}\left[2 \operatorname{Re}\left[\gamma_{\mathcal{C}}^{\mathrm{LO}}\right] \cdot \delta(1-z)+\left(\gamma_{J}^{\mathrm{LO}}(z, \mu)+\gamma_{S}^{\mathrm{LO}}(z, \mu)\right)\right]+\mathcal{O}\left(\alpha_{s}^{2}\right),
\end{aligned}
$$

where $\gamma_{J}$ and $\gamma_{S}$ are the anomalous dimensions for $\bar{J}_{n}$ and $\bar{S}$,

$$
\begin{aligned}
\mu \frac{d}{d \mu} \bar{J}_{n}(x, \mu) & =\int_{x}^{1} \frac{d z}{z} \gamma_{J}(z, \mu) \bar{J}_{n}(x / z, \mu), \\
\mu \frac{d}{d \mu} \bar{S}(x, \mu) & =\int_{x}^{1} \frac{d z}{z} \gamma_{S}(z, \mu) \bar{S}(x / z, \mu) .
\end{aligned}
$$

At the lowest order in $\alpha_{s}$ they are given by

$$
\begin{aligned}
& \gamma_{J}^{\mathrm{LO}}(z, \mu)=\frac{\alpha_{s}}{\pi} C_{F}\left[\delta(1-z)\left(2 \ln \frac{\mu^{2}}{Q^{2}}+\frac{3}{2}\right)-\frac{2}{(1-z)_{+}}\right], \\
& \gamma_{S}^{\mathrm{LO}}(z, \mu)=\frac{\alpha_{s}}{\pi} C_{F}\left[\delta(1-z)\left(-\ln \frac{\mu^{2}}{M^{2}}+1\right)+\frac{2}{(1-z)_{+}}\right] .
\end{aligned}
$$

From (41), (52), and (53) it then follows immediately that Eq. (49) vanishes at $\mathcal{O}\left(\alpha_{s}\right)$.

\section{Resummed result for the differential decay rate near the endpoint}

The factorized result in Eq. (46) still contains large logarithms. We resum these by $\mathrm{RG}$ evolving $\left|\mathcal{C}_{L}\right|^{2}, \bar{J}_{n}$, and $\bar{S}$ from the factorization scale $\mu_{F}$ down to the respective "typical scales" for each of the three quantities. The RG evolution then automatically resums the large logarithms and exponentiates them. Here "the typical scale" denotes the scale at which the logarithms in the expressions for $\left|\mathcal{C}_{L}\right|^{2}, \bar{J}_{n}$, and $\bar{S}$ are minimized. The typical hard scale for $\left|\mathcal{C}_{L}\right|^{2}$ can be chosen as $\mu_{H} \sim M$. On the other hand, Eqs. (47) and (48) imply that we can choose $\mu_{J} \sim M\left(1-r_{\chi}\right)^{1 / 2}(1-z)^{1 / 2}$ and $\mu_{S} \sim M(1-z)$ for the jet and soft functions, respectively.
We perform the resummation to NNLL accuracy, counting large logarithms to be of $O\left(1 / \alpha_{s}\right)$. For resummation to NNLL accuracy, we express the anomalous dimension of each factorized part as follows:

$$
\begin{gathered}
\gamma_{\mathcal{C}}=A_{\mathcal{C}} \Gamma_{C} \ln \frac{\mu^{2}}{M^{2}\left(1-r_{\chi}\right)^{2}}+\hat{\gamma}_{\mathcal{C}}, \\
\gamma_{J}(z)=\delta(1-z)\left[A_{J} \Gamma_{C} \ln \frac{\mu^{2}}{M^{2}\left(1-r_{\chi}\right)}+\hat{\gamma}_{J}\right]-\kappa_{J} \frac{A_{J} \Gamma_{C}}{(1-z)_{+}},
\end{gathered}
$$

$\gamma_{S}(z)=\delta(1-z)\left[A_{S} \Gamma_{C} \ln \frac{\mu^{2}}{M^{2}}+\hat{\gamma}_{S}\right]-\kappa_{S} \frac{A_{S} \Gamma_{C}}{(1-z)_{+}}$.

From Eqs. (41), (52), and (53) we extract $\left\{A_{\mathcal{C}}, A_{J}, A_{S}, \kappa_{J}, \kappa_{S}\right\}=\{-1,2,-1,1,2\} . \Gamma_{C}$ is the cusp anomalous dimension $[35,36]$, which can be expanded as $\sum_{k=0} \Gamma_{k}\left(\alpha_{s} / 4 \pi\right)^{k+1}$. The first two coefficients in the expansion are,

$\Gamma_{0}=4 C_{F}, \quad \Gamma_{1}=4 C_{F}\left[\left(\frac{67}{9}-\frac{\pi^{2}}{3}\right) C_{A}-\frac{10}{9} n_{f}\right]$

where $C_{A}=N_{c}=3$ is the number of colors and $n_{f}$ is the number of flavors. The three loop coefficient $\Gamma_{2}$ reads [37] 


$$
\begin{aligned}
\Gamma_{2}= & 4 C_{F}\left[C_{A}^{2}\left(\frac{245}{6}-\frac{134 \pi^{2}}{27}+\frac{11 \pi^{4}}{45}+\frac{22}{3} \zeta(3)\right)+C_{A} n_{f}\left(-\frac{219}{27}+\frac{20 \pi^{2}}{27}-\frac{18}{3} \zeta(3)\right)\right. \\
& \left.+C_{F} n_{f}\left(-\frac{55}{6}+8 \zeta(3)\right)-\frac{4}{27} n_{f}^{2}\right] .
\end{aligned}
$$

The noncusp anomalous dimensions in Eqs. (55) and (56) can be expanded as $\hat{\gamma}_{f=J, S}=\sum_{k=0} \hat{\gamma}_{f, k}\left(\alpha_{s} /(4 \pi)\right)^{k}$. From Eqs. (52) and (53), the leading coefficients are given as

$$
\hat{\gamma}_{J, 0}=6 C_{F}, \quad \hat{\gamma}_{S, 0}=4 C_{F} .
$$

The two loop coefficients required for NNLL accuracy are given by [38]

$$
\begin{gathered}
\hat{\gamma}_{J, 1}=-2 C_{F}\left[C_{F}\left(-\frac{3}{2}+2 \pi^{2}-24 \zeta(3)\right)+C_{A}\left(-\frac{3155}{54}+\frac{22 \pi^{2}}{9}+40 \zeta(3)\right)+n_{f}\left(\frac{247}{27}-\frac{4 \pi^{2}}{9}\right)+2 \beta_{0}\left(7-2 \pi^{2}\right)\right], \\
\hat{\gamma}_{S, 1}=16 C_{F}\left[C_{A}\left(-\frac{37}{108}+\frac{\pi^{2}}{144}+\frac{9}{4} \zeta(3)-\frac{1}{6}\right)-n_{f}\left(\frac{1}{54}+\frac{\pi^{2}}{72}\right)\right],
\end{gathered}
$$

where $\beta_{0}$ is the first coefficient of QCD beta function. The $\hat{\gamma}_{\mathcal{C}}$ in Eq. (54) can be written as $\hat{\gamma}_{\mathcal{C}}=-\hat{\gamma}_{J}-\hat{\gamma}_{S}$ from the fact that the differential decay width is scale independent.

Performing the RG evolutions using Laplace transform $[39,40]$ leads to the resummed result near the endpoint at NNLL accuracy as

$$
\begin{aligned}
\frac{d \Gamma_{\text {res }}\left(\tilde{q} \rightarrow \chi^{0} X_{q_{L}}\right)}{d z}= & \frac{M}{16 \pi}\left(1-r_{\chi}\right)^{2} \exp \left[\mathcal{M}\left(\mu_{H}, \mu_{J}, \mu_{S}\right)\right]\left|B_{L}\left(\mu_{H}\right)\right|^{2}\left|C_{L}\left(M, r_{\chi}, \mu_{H}\right)\right|^{2} \\
& \times \tilde{J}\left[\ln \frac{\mu_{J}^{2}}{M^{2}\left(1-r_{\chi}\right)}-\partial_{\eta}\right] \tilde{S}\left[\ln \frac{\mu_{S}^{2}}{M^{2}}-2 \partial_{\eta}\right] \frac{e^{-\gamma_{E} \eta}}{\Gamma(\eta)}(1-z)^{-1+\eta}
\end{aligned}
$$

For integrated quantities such as the total decay width the NNLL accuracy requires jet and soft functions, $\tilde{J}$ and $\tilde{S}$, to be calculated at $\mathcal{O}\left(\alpha_{s}\right)$ (see, e.g., Table 1 in Ref. [41]). For decay width distributions, such as $d \Gamma / d z$, the log enhanced $\mathcal{O}\left(\alpha_{s}^{2}\right)$ terms in $\widetilde{J}_{n}$ and $\tilde{S}$ need to be included as well [42] (see, e.g., Table 6 in Ref. [42]). To the required order the two functions are

$$
\begin{aligned}
& \tilde{J}[L]=1+\frac{\alpha_{s} C_{F}}{2 \pi}\left(\frac{7}{2}-\frac{\pi^{2}}{3}+\frac{3}{2} L+L^{2}\right)+\cdots, \\
& \tilde{S}[L]=1+\frac{\alpha_{s} C_{F}}{2 \pi}\left(-\frac{5 \pi^{2}}{12}+L-\frac{1}{2} L^{2}\right)+\cdots
\end{aligned}
$$

where the ellipses denote the $O\left(\alpha_{s}^{2}\right)$ terms, which are given in Appendix B. For the NLL result, we only keep the first two terms in Eq. (63) and (64).

The exponentiation factor in Eq. (62) is given by

$$
\begin{array}{r}
\mathcal{M}\left(\mu_{H}, \mu_{J}, \mu_{S}\right)=2 S_{\Gamma}\left(\mu_{H}, \mu_{J}\right)-2 S_{\Gamma}\left(\mu_{J}, \mu_{S}\right)+\ln \frac{\mu_{H}^{2}}{M^{2}\left(1-r_{\chi}\right)^{2}} a\left[\Gamma_{C}\right]\left(\mu_{H}, \mu_{J}\right) \\
-\ln \frac{\mu_{H}^{2}}{M^{2}} a\left[\Gamma_{C}\right]\left(\mu_{J}, \mu_{S}\right)+a\left[\hat{\gamma}_{J}\right]\left(\mu_{H}, \mu_{J}\right)+a\left[\hat{\gamma}_{S}\right]\left(\mu_{H}, \mu_{S}\right),
\end{array}
$$

with the Sudakov factor $S_{\Gamma}$ and the evolution function $a[f]$ defined as

$$
S_{\Gamma}\left(\mu_{1}, \mu_{0}\right)=\int_{\alpha_{0}}^{\alpha_{1}} \frac{d \alpha}{b(\alpha)} \Gamma_{C}(\alpha) \int_{\alpha_{1}}^{\alpha} \frac{d \alpha^{\prime}}{b\left(\alpha^{\prime}\right)}, \quad a[f]\left(\mu_{1}, \mu_{0}\right)=\int_{\alpha_{0}}^{\alpha_{1}} \frac{d \alpha}{b(\alpha)} f(\alpha)
$$


Here $\alpha$ and $\alpha_{0,1}$ denote $\alpha_{s}(\mu)$ and $\alpha_{s}\left(\mu_{0,1}\right)$, while $b\left(\alpha_{s}\right)=d \alpha_{s} / d \ln \mu$ is the QCD beta function. To NNLL accuracy $S_{\Gamma}$ and $a\left[\Gamma_{C}\right]$ are given by [41]

$$
\begin{aligned}
S\left(\mu_{1}, \mu_{0}\right)= & \frac{\Gamma_{0}}{4 \beta_{0}^{2}}\left\{\frac{4 \pi}{\alpha_{s}\left(\mu_{1}\right)}\left(1-\frac{1}{r}-\ln r\right)+\left(\frac{\Gamma_{1}}{\Gamma_{0}}-\frac{\beta_{1}}{\beta_{0}}\right)(1-r+\ln r)+\frac{\beta_{1}}{2 \beta_{0}} \ln ^{2} r\right. \\
& +\frac{\alpha_{s}\left(\mu_{1}\right)}{4 \pi}\left[\left(\frac{\beta_{1} \Gamma_{1}}{\beta_{0} \Gamma_{0}}-\frac{\beta_{2}}{\beta_{0}}\right)(1-r+r \ln r)+\left(\frac{\beta_{1}^{2}}{\beta_{0}^{2}}-\frac{\beta_{2}}{\beta_{0}}\right)(1-r) \ln r\right. \\
& \left.\left.-\left(\frac{\beta_{1}^{2}}{\beta_{0}^{2}}-\frac{\beta_{2}}{\beta_{0}}-\frac{\beta_{1} \Gamma_{1}}{\beta_{0} \Gamma_{0}}-\frac{\Gamma_{2}}{\Gamma_{0}}\right) \frac{(1-r)^{2}}{2}\right]\right\}, \\
& a\left[\Gamma_{C}\right]\left(\mu_{1}, \mu_{0}\right)=\frac{\Gamma_{0}}{2 \beta_{0}}\left[\ln r+\left(\frac{\Gamma_{1}}{\Gamma_{0}}-\frac{\beta_{1}}{\beta_{0}}\right) \frac{\alpha_{s}\left(\mu_{0}\right)-\alpha_{s}\left(\mu_{1}\right)}{4 \pi}\right],
\end{aligned}
$$

where $r=\alpha_{s}\left(\mu_{0}\right) / \alpha_{s}\left(\mu_{1}\right)$. Finally, the evolution parameter $\eta$ in Eq. (62) is defined as $\eta=2 a\left[\Gamma_{C}\right]\left(\mu_{J}, \mu_{S}\right)$. It is positive since $\mu_{J}>\mu_{S}$. For the NLL result, we only keep the first line of Eq. (67), and the first term in Eq. (68).

\section{DECAY DISTRIBUTION IN THE FULL RANGE}

Even though the decay distribution $d \Gamma / d z$ in the region $z \rightarrow 1$ is the dominant contribution to the total decay width, it is useful for phenomenological analyses to obtain the decay distribution in the full range of $z$, while retaining the $\mathcal{O}(1-z)$ corrections. The expression for $d \Gamma / d z$ away from $z \rightarrow 1$ should be obtained using full QCD. Away from the endpoint region the gluon emissions are hard so that the total invariant mass of final state jets can be comparable to $M$.

To perform the calculation of $\mathcal{T}_{L}$ in Eq. (20) in full QCD we introduce the structure function $W_{L}\left(z, r_{\chi}, M\right)$,

$$
\mathcal{T}_{L}\left(z, r_{\chi}, M\right)=2 \pi\left|B_{L}\right|^{2} W_{L}\left(z, r_{\chi}, M\right)
$$

The $W_{L}$ is thus given by

$$
W_{L}\left(z, r_{\chi}, M\right)=\frac{1}{2 \pi} \int d^{4} z e^{-i p_{\chi} \cdot z}\left\langle\tilde{q}\left|\tilde{q}^{* \alpha}\left(P_{L} q^{\alpha}\right)_{a}(z)\left(\bar{q}^{\beta} P_{R}\right)_{b} \tilde{q}_{L}^{\beta}(0)\right| \tilde{q}\right\rangle\left(p_{\chi}-m_{\chi}\right)_{b a}
$$

where $p_{\chi}$ is the momentum of the neutralino [the expression for it in terms of $z$ and $r_{\chi}$ is given in Eq. (25)]. The differential decay rate is then

$$
\frac{d \Gamma}{d z}\left(\tilde{q} \rightarrow \chi^{0} X_{q_{L}}\right)=\frac{M}{16 \pi}\left|B_{L}(\mu)\right|^{2} \frac{1}{z}\left(z-\frac{r_{\chi}}{z}\right)^{2} W_{L}\left(z, r_{\chi}, M, \mu\right)
$$

At tree level we have simply $W_{L}=\delta(1-z)$.

To obtain the NLO expression for $W_{L}$ we computed the Feynman diagrams shown in Fig. 2 as well as self-energy diagrams for the squark and the light quark. As a result, one loop corrections to $W_{L}$ in $\overline{\mathrm{MS}}$ scheme are given as

$$
\begin{aligned}
W^{(1)}\left(z, r_{\chi}, M, \mu\right)= & \frac{\alpha_{s} C_{F}}{2 \pi}\left\{\delta ( 1 - z ) \left[\frac{3}{2} \ln \frac{\mu^{2}}{M^{2}\left(1-r_{\chi}\right)^{2}}+\frac{1}{4} \ln ^{2}\left(1-r_{\chi}\right)+2 \operatorname{Li}_{2}\left(\frac{r_{\chi}}{r_{\chi}-1}\right)\right.\right. \\
& \left.-2 r_{\chi} H_{1}\left(r_{\chi}\right)-\frac{7}{2} r_{\chi} H_{2}\left(r_{\chi}\right)+\frac{5}{4}-\frac{\pi^{2}}{3}\right]+\frac{r_{\chi}}{z-r_{\chi}}+\frac{1}{(1-z)_{\Delta}} \frac{z}{z-r_{\chi}}\left[-4\left(1-r_{\chi}\right)+\frac{1}{2}\left(z-\frac{r_{\chi}}{z}\right)\right] \\
& \left.-2 \frac{z\left(1-r_{\chi}\right)\left(2-z-r_{\chi} / z\right)}{z-r_{\chi}}\left[g\left(z, r_{\chi}\right)\right]_{\Delta}\right\} .
\end{aligned}
$$

Here we introduced the so called "delta distribution," $[\ldots]_{\Delta}$, in order to deal with the infrared (IR) singularity as $z \rightarrow 1$. The definition and some useful properties of the $\Delta$ distribution are given in Appendix A. For a region of integration that is, as in 


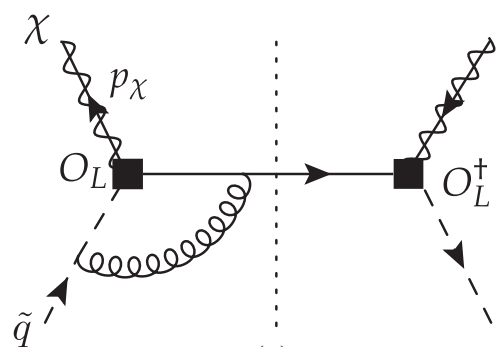

(a)

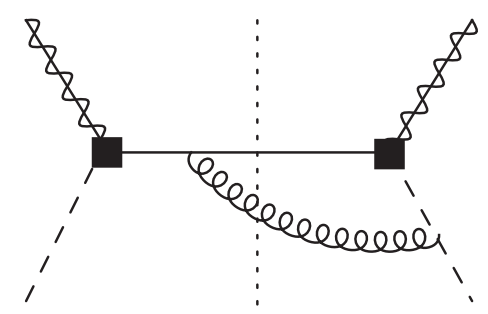

(d)

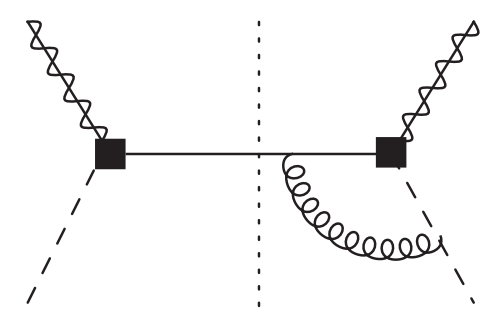

(b)

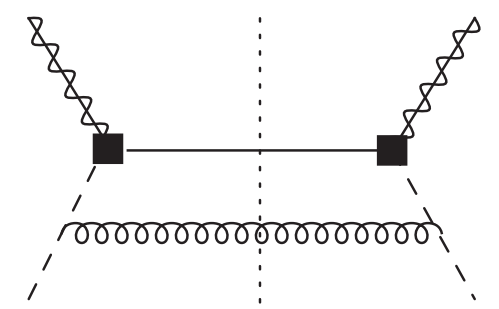

(e)

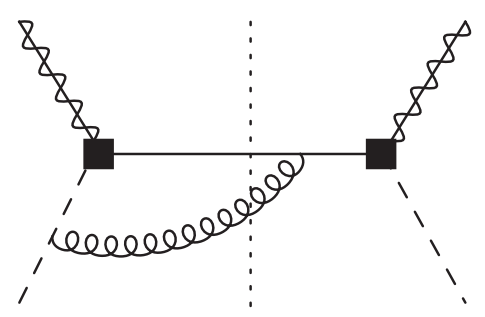

(c)

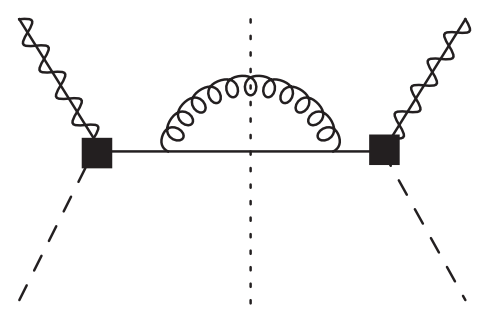

(f)

FIG. 2. The NLO Feynman diagrams for $\tilde{q} \rightarrow \chi X_{q_{L}}$. The dashed lines at the center of each diagram denotes the discontinuity cut for forward scattering amplitudes.

our case, over $z \in\left[\sqrt{r_{\chi}}, 1\right]$ (rather than over the interval $[0,1]$ ) the introduction of a $\Delta$ distribution shortens the expressions compared to the standard plus distribution. In Eq. (72) the functions $H_{1,2}\left(r_{\chi}\right)$ and $g\left(z, r_{\chi}\right)$ are given by,

$$
\begin{gathered}
H_{1}\left(r_{\chi}\right)=\int_{\sqrt{r_{\chi}}}^{1} \frac{d z}{z} \frac{\left(2-r_{\chi}-r_{\chi} / z\right)\left(1-2 r_{\chi} / z+r_{\chi} / z^{2}\right)}{\left(z-r_{\chi} / z\right)\left(1-r_{\chi} / z\right)^{2}} \ln \frac{1-z}{1-r_{\chi} / z}, \\
H_{2}\left(r_{\chi}\right)=\int_{\sqrt{r_{\chi}}}^{1} \frac{d z}{z} \frac{1-2 r_{\chi} / z+r_{\chi} / z^{2}}{\left(1-r_{\chi} / z\right)^{2}}=\frac{1}{\sqrt{r_{\chi}}}+2 \operatorname{coth}^{-1}\left(1+2 \sqrt{r_{\chi}}\right)-\frac{\ln \left(1+\sqrt{r_{\chi}}\right)}{x},
\end{gathered}
$$

$$
g\left(r_{\chi}, z\right)=\frac{z}{(1-z)\left(z^{2}-r_{\chi}\right)} \ln \frac{1-z}{1-r_{\chi} / z}
$$

The anomalous dimension $\gamma_{W}(z)$, controlling the RG evolution of structure functions,

$\mu \frac{d}{d \mu} W_{L}\left(M, r_{\chi}, z, \mu\right)=\int_{z}^{1} \frac{d x}{x} \gamma_{W}(x) W_{L}\left(M, r_{\chi}, z / x, \mu\right)$

is given by

$$
\gamma_{W}(z)=3 \frac{\alpha_{s} C_{F}}{2 \pi} \delta(1-z)+\mathcal{O}\left(\alpha_{s}^{2}\right)
$$

Using the fact that $d \Gamma / d z$ is scale-invariant then gives the anomalous dimension for $B_{L}(\mu)$ as $\gamma_{B}=-3 \alpha_{s} C_{F} /(2 \pi)+$ $\mathcal{O}\left(\alpha_{s}^{2}\right)$, which, as expected, is the same result as given in Eq. (42).
Finally, we combine our results for the differential decay rates in the full $z$ range and near the endpoint, $z \rightarrow 1$, to obtain the decay distribution at NNLL $+\mathrm{NLO}^{2}$

\footnotetext{
${ }^{2}$ One may wish to consider other matching schemes that turn off smoothly the resummation effects in the region far away from the endpoint. In our case the role of the smooth matching is performed by the running of jet and soft scales which are respectively given as $\mu_{J}^{0}=M \sqrt{\left(1-r_{\chi}\right)(1-z)}$ and $\mu_{S}^{0}=$ $M(1-z)$, where $r_{\chi}=m_{\chi}^{2} / M^{2}$. While the prescription in Eq. (78) does not recover exactly the NLO result anywhere in the physical region $z \geq \sqrt{r_{\chi}}$, this treatment does suffice for our purposes. First of all, alternative prescriptions that have $\mu_{H}=$ $\mu_{J}=\mu_{S}$ at $z=0$ similarly do not lead to perfectly smooth matchings anywhere in the physical region for $z$. More importantly, the two decay distributions with and without resummations, $d \Gamma_{\text {res }} / d z$ and $d \Gamma_{\mathrm{NLO}}^{f} / d z$, are completely dominated by the endpoint region and have only negligible contributions from the rest of the $z$ range. We therefore expect only numerically subleading corrections from alternative matching prescriptions relative to the results using Eq. (78).
} 
TABLE I. Total decay widths of a $1.45 \mathrm{TeV}$ squark based on the LO, fixed-order (NLO), NLL + NLO, and NNLL + NLO calculations, with the Wilson coefficient $B_{L}$ at the scale $M$ taken to be $B_{L}(M)=1$. Benchmark neutralino masses are varied in the interval $200<M_{\chi}<1000 \mathrm{GeV}$. The impact of scale uncertainties for NLL + NLO and NNLL + NLO predictions are shown as well.

\begin{tabular}{lccccrc}
\hline \hline$M_{\chi}(\mathrm{GeV})$ & LO $(\mathrm{GeV})$ & $\mathrm{NLO}(\mathrm{GeV})$ & NLL+NLO $(\mathrm{GeV})$ & unc. $(\%)$ & NNLL+NLO $(\mathrm{GeV})$ & unc. $(\%)$ \\
\hline 1000 & 7.93 & 7.87 & 7.45 & \pm 18.13 & 8.58 & \pm 14.81 \\
900 & 10.90 & 10.85 & 9.92 & \pm 17.79 & 11.47 & \pm 14.20 \\
800 & 13.96 & 13.90 & 12.40 & \pm 17.76 & 14.38 & \pm 13.94 \\
700 & 16.97 & 16.91 & 14.79 & \pm 17.90 & 17.19 & \pm 13.90 \\
600 & 19.81 & 19.74 & 17.01 & \pm 18.13 & 19.81 & \pm 13.98 \\
500 & 22.39 & 22.30 & 18.98 & \pm 18.39 & 22.15 & \pm 14.12 \\
400 & 24.62 & 24.50 & 20.65 & \pm 18.66 & 24.14 & \pm 14.29 \\
300 & 26.43 & 26.29 & 22.00 & \pm 18.91 & 25.73 & \pm 14.46 \\
200 & 27.76 & 27.62 & 23.23 & \pm 18.93 & 27.16 & \pm 14.49 \\
\hline \hline
\end{tabular}

$$
\frac{d \Gamma_{\text {full }}}{d z}\left(\tilde{q}_{L} \rightarrow \chi^{0} X_{q}\right)=\frac{d \Gamma_{\text {res }}}{d z}+\frac{d \Gamma_{\mathrm{NLO}}^{f}}{d z}-\frac{d \Gamma_{E}^{f}}{d z} .
$$

The first and the second terms on the right-hand side are the resummed result near the endpoint, Eq. (62), and the NLO result for the full $z$ range, Eq. (71), respectively. The double counting of contributions between the two terms is removed by the third term on the right-hand side of Eq. (78), i.e., the $d \Gamma_{E}^{f} / d z$. The expression for $d \Gamma_{E}^{f} / d z$ follows from $d \Gamma_{\text {res }} / d z$ by identifying the multiple scales as $\mu_{H}=\mu_{J}=\mu_{S}=M$.

\section{PHENOMENOLOGICAL STUDY}

In this section, we present a detailed study of NLO, NLL + NLO, and NNLL + NLO predictions for the $\tilde{q} \rightarrow q \chi$ decay. In Sec. VA we show our results for the normalized differential width distributions as well as the total decay widths, and discuss the impact of soft gluon resummations on the $\mathrm{NLL}+\mathrm{NLO}$ and NNLL $+\mathrm{NLO}$ results. In Sec. V B, we perform a numerical analysis at $\mathrm{NLL}+\mathrm{NLO}$ and NNLL + NLO accuracies for the decays of pair-produced squarks in a linear $e^{+} e^{-}$collider based on $\sqrt{s}=3 \mathrm{TeV}$ Compact Linear Collider (CLIC). Since the decay topology of a squark can be significantly altered by higher-order corrections, it is necessary to scrutinize these effects for the precise measurements of a squark and neutralino masses, which is an important part of the CLIC physics program.

\section{A. Differential width distributions and total widths}

The resummed results that we calculated at NNLL + NLO and NLL + NLO accuracies in Eq. (62) depend on the choices of scales, $\mu_{H}, \mu_{J}$, and $\mu_{S}$. To illustrate the scale dependences, we independently vary the $\mu_{i}, i=H, J, S$, between $2 \mu_{i}^{0}$ and $\mu_{i}^{0} / 2$, where $\mu_{i}^{0}$ are the default choices of the hard, jet, and soft scales. We take $\mu_{H}^{0}=M$ for the default hard scale. The default jet and soft scales are chosen as the running scales $\mu_{J}^{0}=M \sqrt{\left(1-r_{\chi}\right)(1-z)}$ and
$\mu_{S}^{0}=M(1-z)$, where $r_{\chi}=m_{\chi}^{2} / M^{2}$. For $z \rightarrow 1$ we would have $\mu_{i}^{0} \rightarrow 0$ for these choices of running scales and therefore the IR Landau poles in the running of the strong coupling constants. In order to avoid this problem we adopt the following profile function for the soft scale,

$$
\mu_{S}^{0}=\mu_{S}^{p f}= \begin{cases}M(1-z) & \text { if } z \leq z_{0} \\ \mu_{\min }+a M(1-z)^{2} & \text { if } z>z_{0}\end{cases}
$$

Therefore we make the soft scale frozen as $\mu_{\min }$ as $z \rightarrow 1$. The parameters $a$ and $z_{0}$, where $1-z_{0} \ll 1$, are determined by $z_{0}=1-2 \mu_{\min } / M$ and $a=M /\left(4 \mu_{\min }\right)$ to ensure that $\mu_{S}^{p f}$ is smoothly continuous at $z_{0}$. We use $\mu_{\text {min }}=0.5 \mathrm{GeV}$ so that $M\left(1-z_{0}\right)=1 \mathrm{GeV}$. The impact of nonperturbative physics grows as $z$ becomes larger than $z_{0}$, and therefore $\mu_{S}^{0}$ goes from $1 \mathrm{GeV}$ to $0.5 \mathrm{GeV}$. The precise choice of $\mu_{\min }$ and the estimation of its uncertainty would be possible from a nonperturbative model or from a fit to experimental data, if these become available. This is beyond the scope of this paper, where we focus on perturbative resummation effects near the endpoint.

We also modify the jet scale using the following profile function $^{3}$

$$
\mu_{J}^{0}=\mu_{J}^{p f}=\sqrt{M\left(1-r_{\chi}\right) \mu_{S}^{p f}} .
$$

Table I shows the total decay widths of a squark with mass $M=1.45 \mathrm{TeV}$ obtained at LO, NLO, NLL + NLO, and NNLL + NLO accuracies, with the Wilson coefficient $B_{L}$ normalized by $B_{L}(M)=1$ at the scale $M$. Benchmark neutralino masses are chosen in the interval $200<M_{\chi}<$ $1000 \mathrm{GeV}$. The scale uncertainty of NLL + NLO prediction turns out to be $\sim 18 \%$, which is improved to $\sim 14 \%$ at

\footnotetext{
${ }^{3}$ Equation (80) relates only the default jet and soft scales. When we estimate the uncertainty due to the choice of scales, the jet and soft scales are varied independently around these central values.
} 

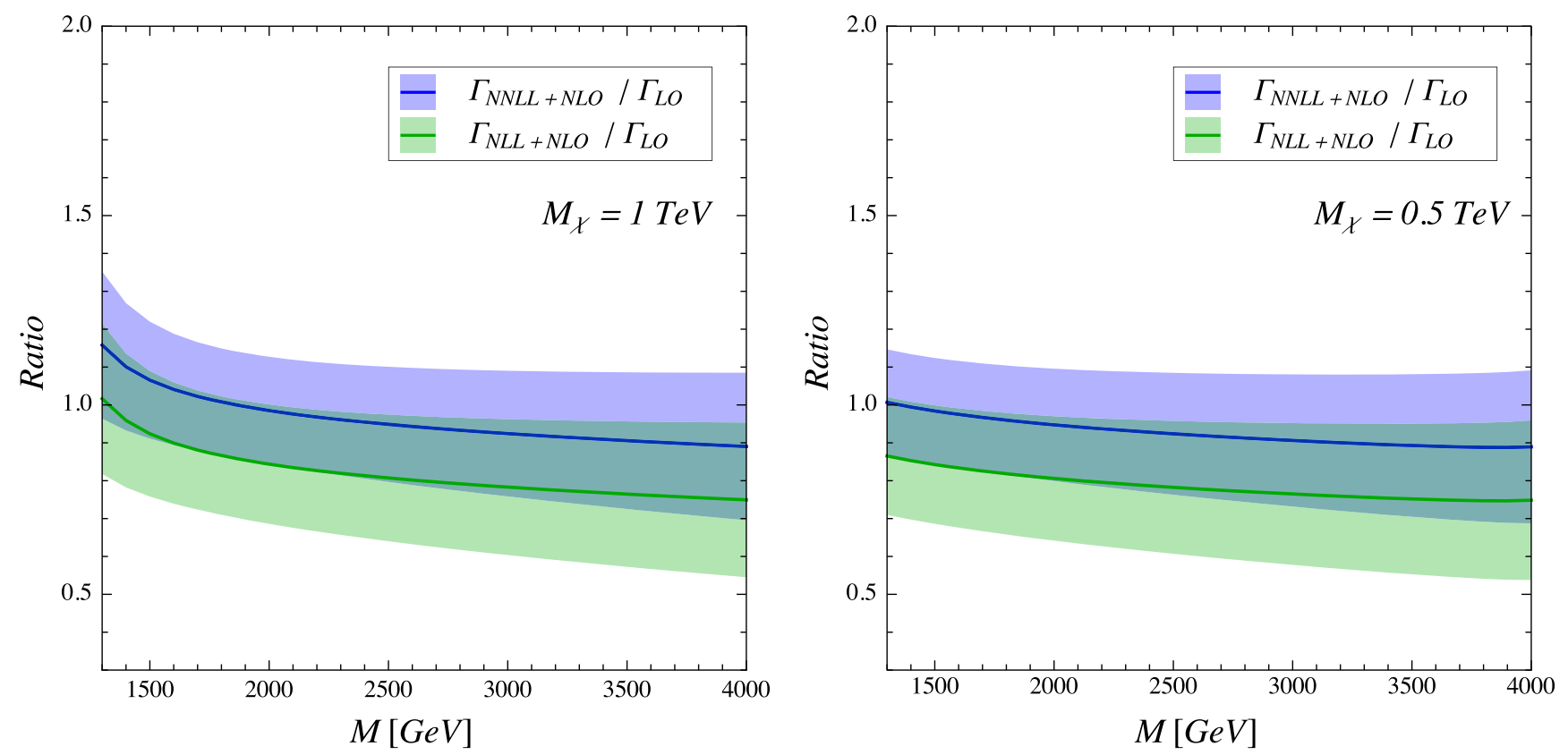

FIG. 3. The ratios of NLL + NLO (green line) and NNLL + NLO (blue) total decay widths normalized to the LO result as a function of mass $M$. The neutralino masses are fixed to $M_{\chi}=1(0.5) \mathrm{TeV}$ in the left (right) panel. The details on hard, jet, and soft scale variations, giving the corresponding bands, are explained in Eq. (79) and Eq. (80), with $\mu_{\min }=0.5 \mathrm{GeV}$.

NNLL + NLO. These are obtained by varying the hard, jet and soft scales in the range from $\mu_{H, J, S}^{0} / 2$ to $2 \mu_{H, J, S}^{0}$ each independently. Figure 3 shows the ratios of total decay widths of NLL + NLO and NNLL + NLO predictions with respect to the $\mathrm{LO}$ as a function of mass $M$, while fixing (left)
$M_{\chi}=1 \mathrm{TeV}$ and (right) $M_{\chi}=0.5 \mathrm{TeV}$. For both cases, scale uncertainties become larger as $M$ increases.

Normalized NLL + NLO, NNLL + NLO, and NLO differential decay width distributions using $\mu_{\min }=$ $0.5 \mathrm{GeV}$ are shown in Figure 4. The NLO distribution

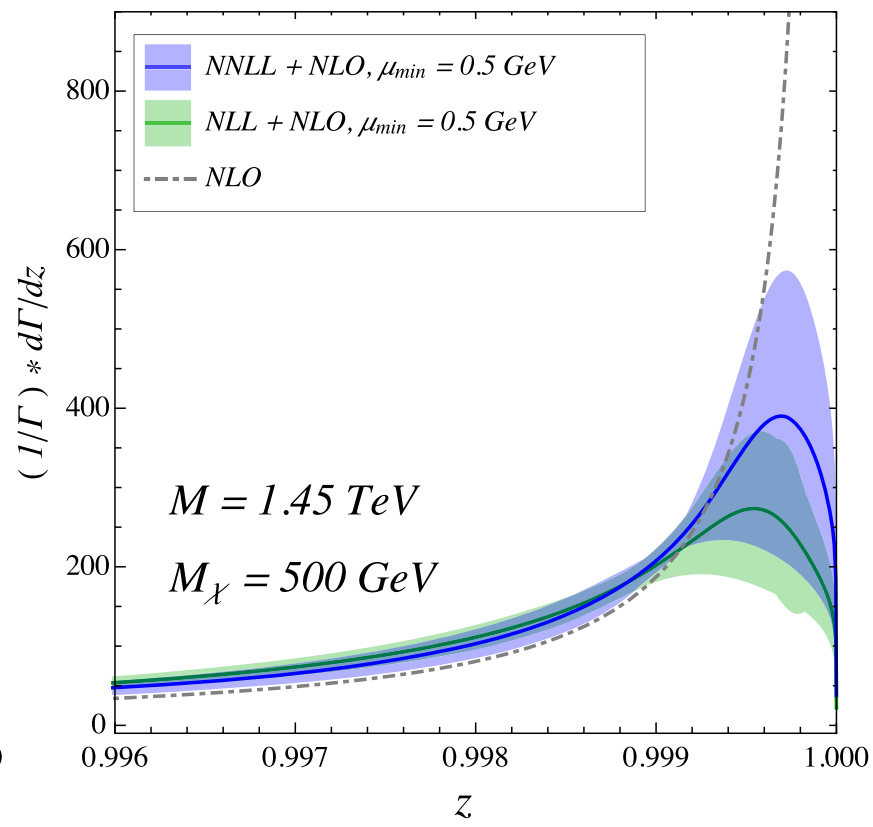

FIG. 4. Normalized NLL + NLO (green), NNLL + NLO (blue), and NLO (gray dash-dotted) differential decay width distributions, using $\mu_{\min }=0.5 \mathrm{GeV}$. We fixed neutralino masses to (left panel) $M_{\chi}=1 \mathrm{TeV}$ and (right panel) $M_{\chi}=0.5 \mathrm{TeV}$. 
diverges in the region $z \rightarrow 1$, while the resummed NLL + NLO and NNLL + NLO distributions are regulated at the endpoint. The central value of NLL + NLO distribution tends to be more broadened compared to the NNLL + NLO, but their overall size of uncertainties near the endpoint region are similar with each other.

Concerning the scale variations of the NLL + NLO and NNLL + NLO distributions, we find that the dominant uncertainties come from when we vary the soft scale down to $\mu_{S}^{0} / 2$, while other scale variations give quite small uncertainties. Note that $\mu_{S}^{0}$ varies from $5.8 \mathrm{GeV}$ to $1.45 \mathrm{GeV}$ when $z$ is changed from $z=0.996$ to $z=0.999$, and reaches $\mu_{\min }=0.5 \mathrm{GeV}$ at the very right end of panels in Fig. 4. The large variation still present at NNLL for $z>0.999$ can thus be traced to this very low soft scale that is reached at the very endpoint of the spectrum.

\section{B. Precision studies of squarks and neutralinos at CLIC}

The squark decay, $\tilde{q} \rightarrow q \chi$, results in a two-body final state at LO, which at higher orders becomes a multibody final state due to additional hard or soft QCD radiation. This can potentially affect the methods for precise measurements of squark and neutralino masses. As an illustration we take the impact of QCD corrections on such measurements at CLIC [43-46], a future linear $e^{+} e^{-}$ collider designed to provide collision energies up to $3 \mathrm{TeV}$. If supersymmetric particles are light enough to be produced at such machine, CLIC will provide a platform for precision studies where their properties could be determined with considerable accuracy [47-51]. In the phenomenological analysis we focus exclusively on the impact of QCD radiations in the squark decay. For a realistic study other important effects, in particular the initial state QED radiation, that results in the reduced effective $e^{+} e^{-}$collision energy, need to be included.

For pair-produced squarks that decay into light quarks and neutralinos,

$$
e^{+} e^{-} \rightarrow \tilde{q} \tilde{q}^{*} \rightarrow q \chi \bar{q} \chi,
$$

an interesting technique to simultaneously measure squark and neutralino masses, is to search for the edges in the event distributions. We will discuss two such methods, (i) based on edges in energy distribution of the light quark jets, $E_{1}+E_{2}$, and (ii) a method based on the kinematic variable $M_{C}$ [52]. The numerical analysis is based on LO version of MadG5_aMC@NLO [53,54] with PYTHIA 6 [55] showering, but no hadronization, which was used to generate the event chain in (81), utilizing the minimal supersymmetric Standard Model (MSSM) implementation from [56,57]. The PYTHIA events were clustered with the FASTJET [58] implementation of the anti- $k_{T}$ algorithm [59], taking $r=0.4$ for the cone size. For events to pass the selection cuts we require at least two jets with $p_{T}>50 \mathrm{GeV}$. To obtain the NLL + NLO and $\mathrm{NNLO}+\mathrm{NLO}$ (including two-loop-log terms) samples we reweight PYTHIA events, on an event-by-event basis, according to the $d \log \Gamma / d z$ normalized differential distributions in Figure 4 for each of the decay chains. We first rewrite the variable $z$ in Lorentz-invariant form

$$
z=\frac{x+\sqrt{x^{2}-4 r_{\chi}}}{2} \quad \text { with } \quad x=2 \frac{p_{\chi} \cdot p_{\tilde{q}}}{M^{2}},
$$

where $p_{\tilde{q}}$ and $p_{\chi}$ are four-momenta of a squark and a neutralino respectively. ${ }^{4}$ We plug two $z$ values (from two decay chains) into the normalized NLL + NLO and $\mathrm{NNLO}+\mathrm{NLO}$ distributions in Fig. 4 using $\mu_{\min }=$ $0.5 \mathrm{GeV}$ to obtain probabilities. Then we multiply the two probabilities to obtain the weight for each event. In this way, the simulated events acquire the correct NLL + NLO and $\mathrm{NNLO}+\mathrm{NLO}$ distributions in $z$ variable, but are only approximately NLL + NLO and NNLO + NLO in the other phase space variables. The NLO samples, on the other hand, cannot be obtained using the same reweighting method. Since the NLO distributions in Fig. 4 diverge at $z=1$, the probabilistic interpretation of the differential width distributions is not well-defined. As a result we do not include reweighted NLO distributions. We derive results for two benchmarks, setting squark mass to $M=1.45 \mathrm{TeV}$, while taking the lightest neutralino mass to be $M_{\chi}=1 \mathrm{TeV}$ or $0.5 \mathrm{TeV}$, and assume a negligible squark decay width. In this study, the beamstrahlung, initial state radiation, and detector effects are not included.

For two body squark decays, (81), the minimal and maximal light quark energy are directly related to $M$ and $M_{\chi}[60,61]$

$$
\begin{aligned}
& E_{q, \max }=\frac{\sqrt{s}}{4}\left(1-\frac{M_{\chi}^{2}}{M^{2}}\right)\left(1+\sqrt{1-\frac{4 M^{2}}{s}}\right), \\
& E_{q, \min }=\frac{\sqrt{s}}{4}\left(1-\frac{M_{\chi}^{2}}{M^{2}}\right)\left(1-\sqrt{1-\frac{4 M^{2}}{s}}\right),
\end{aligned}
$$

and thus in our case $E_{1}+E_{2} \in\left[2 E_{q, \min }, 2 E_{q, \max }\right]$, neglecting the small squark boosts in the lab frame. At LO the $E_{1}+E_{2}$ distributions start at $0.59 \mathrm{TeV}$ and $0.98 \mathrm{TeV}$, for $M_{\chi}=0.5 \mathrm{TeV}$ and $M_{\chi}=1 \mathrm{TeV}$, respectively. In Figure 5, on the other hand, the NLL + NLO and NNLL + NLO $E_{1}+E_{2}$ distributions extend well below these boundaries (see the green and blue lines). This behavior is easy to understand - the collinear radiation leads to nonzero jet masses, or equivalently, to a $d \log d \Gamma / d z$ squark decay distribution with most of the events having $z<1$ (twobody decays have $z=1$ ), see Fig. 4. This in turn means that the jet energy is smaller than in the two body decay, cf. (25), softening the $E_{1}+E_{2}$ spectrum. The effect is

\footnotetext{
${ }^{4}$ We keep track of the event history to access this information.
} 

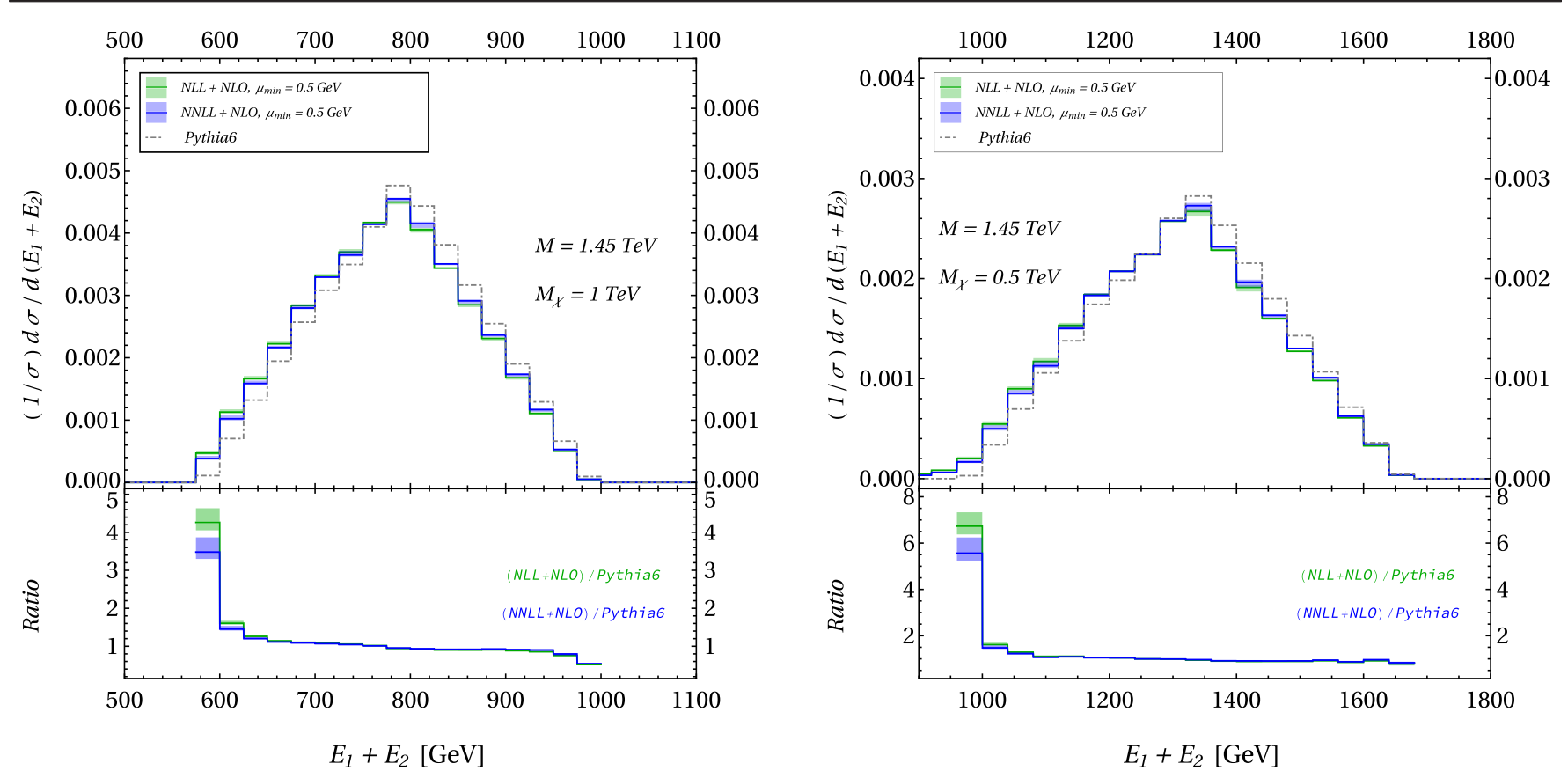

FIG. 5. Distributions for the sum of energies of the first two hardest jets for $M_{\chi}=1 \mathrm{TeV}$ (left) and $M_{\chi}=500 \mathrm{GeV}$ with fixed $M=1.45 \mathrm{TeV}$ (right).

present, but less pronounced, also at the upper edge of the $E_{1}+E_{2}$ distribution. The original PYTHIA distributions (before reweighting) are shown with gray lines.

The extraction of $M$, and $M_{\chi}$ from the $E_{1}+E_{2}$ distribution is still possible, as indicated by the fact that the $E_{1}+E_{2}$ distributions shifts significantly between the $M_{\chi}=0.5 \mathrm{TeV}$ and $M_{\chi}=1 \mathrm{TeV}$ benchmarks. However, one would need to use the full matrix element and not just the edges, in this way controlling the shift of the edges due to the soft and collinear radiations. In addition to the NLL + NLO and NNLL + NLO decay width distributions that we have calculated in the present manuscript, one would also control other systematics and theoretical uncertainties. The method, for instance, requires precise knowledge of the center of mass energy, which can be potentially distorted by beamstrahlung $[62,63]$ and initial state radiations (ISR), causing sizable uncertainties in the measurements of the edges, see, e.g., Ref. [61].

An alternative mass measurement method exploits the kinematic variable $M_{C}$, invariant under contralinear boosts of equal magnitude,

$$
M_{C}=\sqrt{\left(E_{q, 1}+E_{q, 2}\right)^{2}-\left(\vec{p}_{q, 1}-\vec{p}_{q, 2}\right)^{2}}
$$

Here $E_{q, 1}, \vec{p}_{q, 1}$ and $E_{q, 2}, \vec{p}_{q, 2}$ are the energies and threemomenta of the two final state quarks, respectively. The maximal value of $M_{C}$ is reached when the two jets are colinear. It is given by

$$
M_{C}^{\max }=\frac{M^{2}-M_{\chi}^{2}}{M},
$$

showing that $M_{C}$ is sensitive to both $M$ and $M_{\chi}$. The virtue of the $M_{C}$ variable is that it does not depend on the center of mass energy, and is therefore less susceptible to beamstrahlung distortions [61]. Similarly to the $E_{1}+E_{2}$ distribution, the collinear and soft radiations cause the $M_{C}$ spectrum to soften. However, as can be seen in Fig. 6, the effect is more pronounced at the maximal value of $M_{C}$, which is exactly the quantity that enters the determination of $M$ and $M_{\chi}$. Comparing the shift in the distributions for $M_{\chi}=0.5 \mathrm{TeV}$ and $M_{\chi}=1 \mathrm{TeV}$ one sees that the LO sensitivity to $M, M_{\chi}$, Eq. (86), still applies to a good extent also to the resummed distribution with $M_{C}$ constructed using the two hardest jets. For instance, for the numerical examples in Fig. 6 there are still appreciable numbers of events within $\mathcal{O}(5 \%)$ of $M_{C}^{\max }$, with the peak of the distribution shifted by $\mathcal{O}(10-20 \%)$ at NNLL + NLO compared to PYTHIA. This gives a rough sense of associated errors on $M_{C}^{\max }$ due to the softening of distributions in the case of limited statistics available in an experiment. However, once CLIC collects enough statistics a precise determination of $M_{C}^{\max }\left(M, M_{\chi}\right)$ using a matrix element method based on resummed distributions can be attempted.

Finally, we show in Fig. 7 the PYTHIA (gray), NLL + NLO (green), and NNLL + NLO (blue) missing energy $E^{\text {miss }}$ distributions. Here the $E^{\text {miss }}$ is due to the two neutralinos in the final state, and we do not include any detector effect. Unlike the other two observables, $E_{1}+E_{2}$ and $M_{C}$, the effect of resummations is negligible for the 

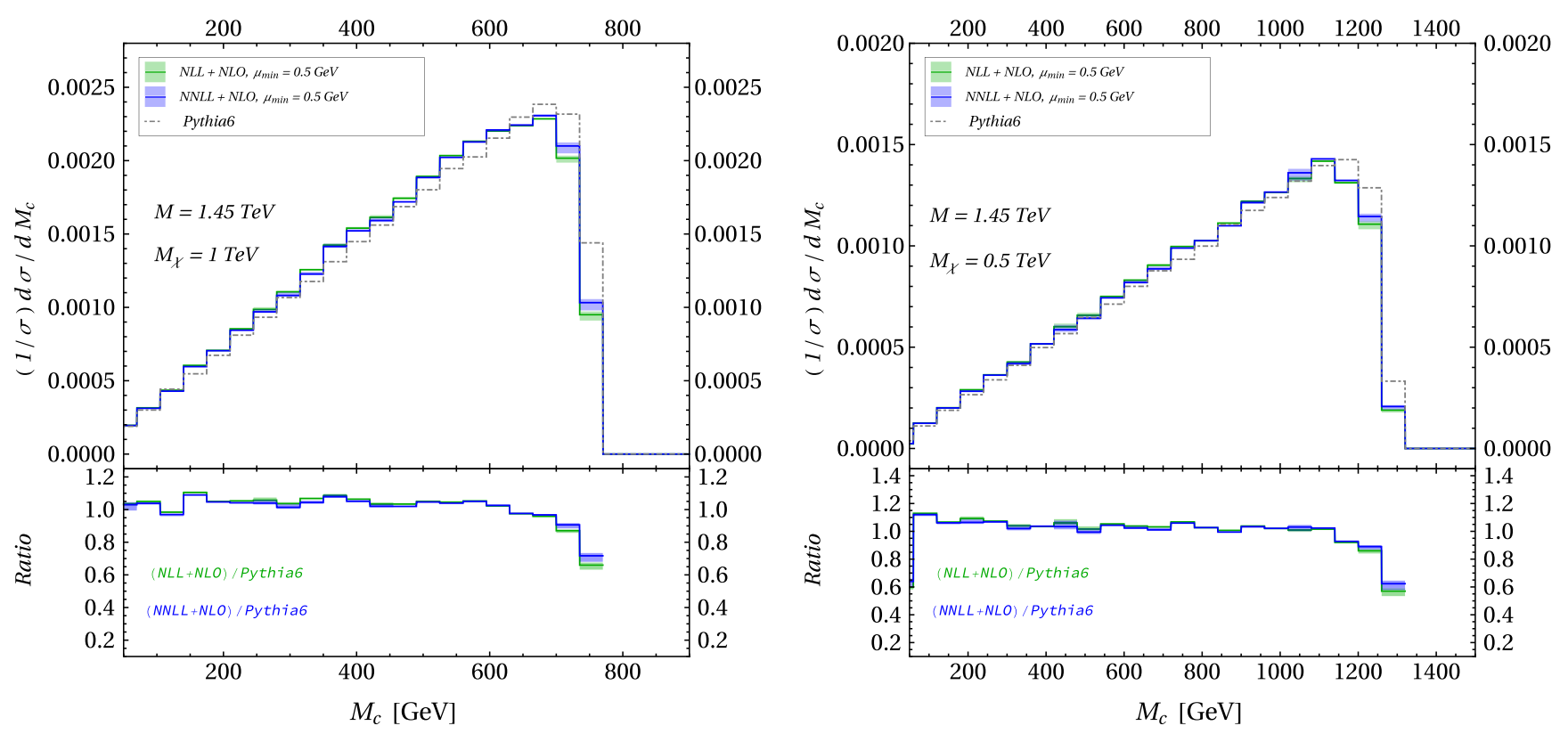

FIG. 6. The $M_{C}$ distributions for (left) $M_{\chi}=1 \mathrm{TeV}$ and (right) $M_{\chi}=500 \mathrm{GeV}$ with fixed $M=1.45 \mathrm{TeV}$.
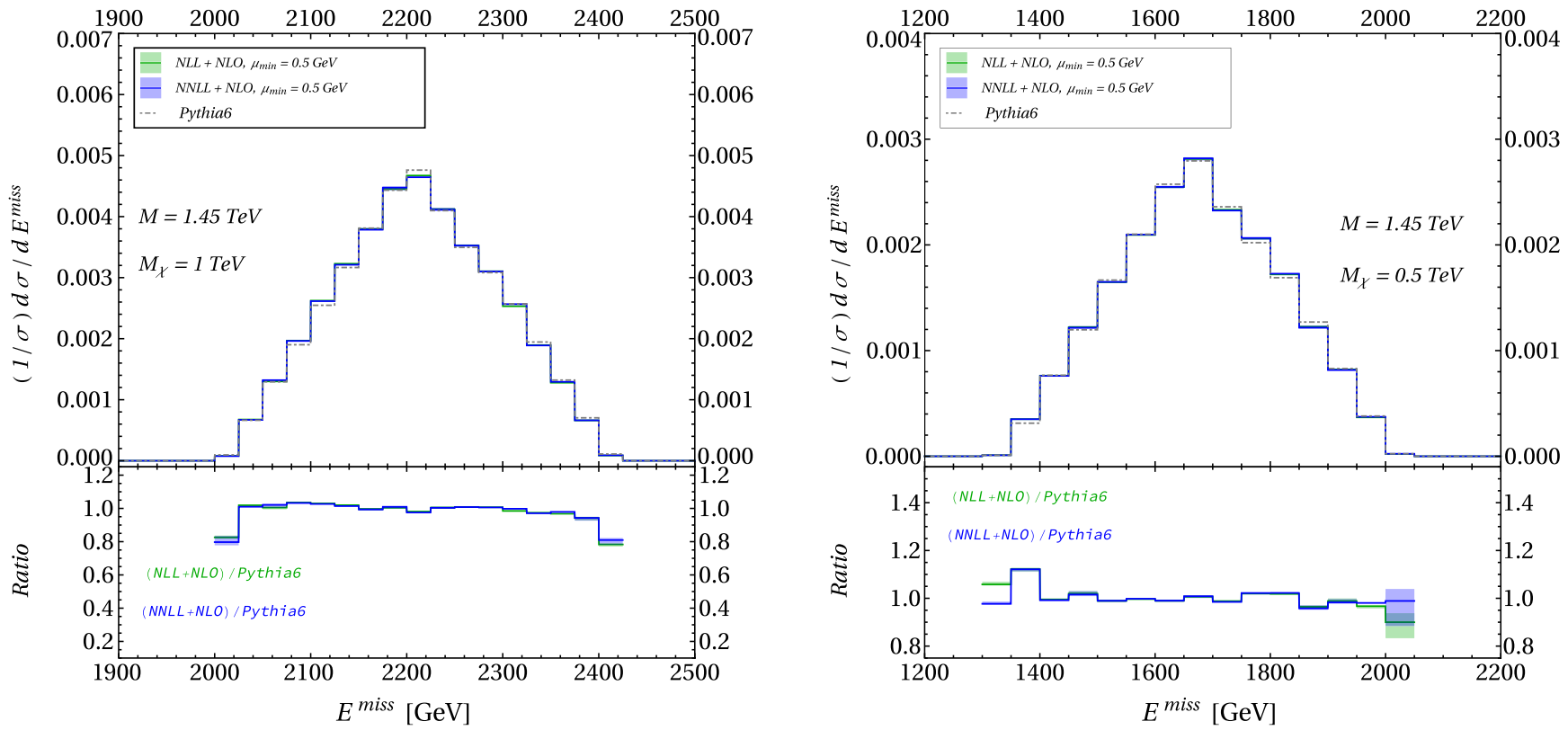

FIG. 7. The missing energy distributions for (left) $M_{\chi}=1 \mathrm{TeV}$ and (right) $M_{\chi}=500 \mathrm{GeV}$ with fixed $M=1.45 \mathrm{TeV}$.

$E^{\text {miss }}$ distribution. This is because the neutralino mass is too heavy for the effect of recoiling against the soft gluon radiations from the quark-sector to be significant.

\section{SUMMARY}

In this paper we have studied QCD corrections to the squark decay, $\tilde{q} \rightarrow q \chi$. The large logarithms that arise in the endpoint region, $z \rightarrow 1$, were resummed using SCET up to the NNLL accuracy. Away from the endpoint we computed hard gluon radiations at NLO. Finally, we provided an expression that smoothly interpolates between the NNLL and NLO results, giving the NNLL + NLO prediction for the total decay width and the decay distribution, $d \Gamma / d z$. The additional QCD radiation in the decay softens the decay distributions for many observables. As a case study for the phenomenological impact of higher order QCD corrections we explored the methods for simultaneous 
measurements of squark and neutralino masses at a linear $e^{+} e^{-}$collider based on $\sqrt{s}=3 \mathrm{TeV}$ CLIC. A majority of mass measurement techniques are based on edges in kinematic distributions. Such kinematic edges are modified by having additional QCD radiation in the event. For instance, the distribution of the combined energy of the hardest two jets, $E_{1}+E_{2}$, now extends below the lower boundary that is otherwise obtained in the case of two body decays. Similarly, the distributions in the $M_{C}$ variable get softened near its maximal value, which is precisely the region used for the quark and neutralino mass extractions. With limited available statistics in experiments this softening of the distributions would result in a shift in measured squark and neutralino masses. The induced shift in the masses could be estimated from a matrix element based method using the NNLL + NLO resummed decay distributions that we provided. In a quantitative analysis one would also need to include additional effects such as the beamstrahlung, initial state radiation, and detector effects.

\section{ACKNOWLEDGMENTS}

J. H. K. is grateful to Kyongchul Kong and Ian M. Lewis for useful discussions and suggestions during the course of this project. C. K. is supported by Basic Science Research Program through the National Research Foundation of Korea (NRF) funded by the Ministry of Science and ICT (Grant No. NRF2017R1A2B4010511). J. H. K. was supported in part by United States Department of Energy Grant No. DE-SC0017988 and by the University of Kansas General Research Fund allocation 2302091. J. H. K. acknowledges the support in part by National Science Foundation under Grant No. PHY1820860 and No. PHY1230860. S. L. was supported by Basic Science Research Program through the National Research Foundation of Korea(NRF) funded by the Ministry of Education (Grant No. NRF-2018R1D1A1B07049148), by the Korea government (MEST) (Grant No. NRF2015R1A2A1A15052408). J.Z. acknowledges support in part by the DOE Grant No. de-sc0011784.

\section{APPENDIX A: DETAILS ABOUT $\Delta$-DISTRIBUTIONS}

Consider function $g(u, \epsilon)$ that is singular at $\epsilon=0$ with $u=0$, and define

$$
A(\epsilon)=\int_{0}^{1} \operatorname{dug}(u, \epsilon),
$$

where $A(\epsilon)$ can have $(1 / \epsilon)^{n}$ poles. The $g(u, \epsilon)$ function can be rewritten in terms of delta function and plus distributions as,

$$
g(u, \epsilon)=A(\epsilon) \delta(u)+[g(u, \epsilon)]_{+},
$$

where the plus distribution is defined as

$$
\int_{0}^{1} d u[g(u)]_{+} f(u)=\int_{0}^{1} d u g(u)[f(u)-f(0)] .
$$

For application to our results it is useful to make a change of variables, $u=h(z)$, so that the singular point, $u=0$, is now at $z=1$, while $z \in[a, 1]$, i.e., $h(1)=0$ and $h(a)=1$. When extracting the IR poles from the integral over $z$ one needs to carefully keep track all the factors due to a change of variables. Consider now the integral

$$
\begin{aligned}
\int_{a}^{1} d z f(z) g(u, \epsilon) \\
\quad=\int_{0}^{1} d u\left(-\frac{d z}{d u}\right) \tilde{f}(u)\left(A(\epsilon) \delta(u)+[g(u, \epsilon)]_{+}\right),
\end{aligned}
$$

where on the lhs $g(u, \epsilon)=g(h(z), \epsilon)$, while on the rhs $\tilde{f}(u)=f\left(h^{-1}(u)\right)=f(z)$. Using Eq. (A3) we can rewrite the above expression as

$$
\begin{aligned}
& \int_{a}^{1} d z f(z) g(u, \epsilon) \\
& =\int_{0}^{1} d u\left(-\frac{d z}{d u}\right) \tilde{f}(u) A(\epsilon) \delta(u) \\
& \quad-\int_{0}^{1} d u g(u, \epsilon)\left(\frac{d z}{d u} \tilde{f}(u)-\left.\frac{d z}{d u}\right|_{u=0} \tilde{f}(0)\right) \\
& =\int_{a}^{1} d z f(z) A(\epsilon) \delta(1-z) /\left|\frac{d u}{d z}\right|_{z=1} \\
& \quad+\int_{a}^{1} d z g(u, \epsilon)\left[f(z)-f(1) \frac{d u}{d z} /\left(\left.\frac{d u}{d z}\right|_{z=1}\right)\right],
\end{aligned}
$$

where in the last two lines $u$ should be viewed as a function of $z, u=h(z)$. This means that in terms of the $z$-space distributions we have

$$
g(u, \epsilon)=A(\epsilon)\left|\frac{d u}{d z}\right|_{z=1}^{-1} \delta(1-z)+[g(u, \epsilon)]_{\Delta},
$$

where we have defined $\Delta$-distribution as

$$
\begin{aligned}
& \int_{a}^{1} d z f(z)[g(u, \epsilon)]_{\Delta} \\
& \quad=\int_{a}^{1} d z g(u, \epsilon)\left[f(z)-f(1) \frac{d u}{d z} /\left(\left.\frac{d u}{d z}\right|_{z=1}\right)\right] .
\end{aligned}
$$

Note that term in the bracket multiplying $g(u, \epsilon)$ in the right side tends to zero as $z \rightarrow 1$.

In obtaining Eq. (72) in the main text, we used the change of variables, $u=(1-z) /(1-x / z)$, for which $d u / d z=$ $-\left(1-2 x / z+x / z^{2}\right) /(1-x / z)^{2}$, and $d u /\left.d z\right|_{z=1}=-1 /$ $(1-x)$. A function $g(u, \epsilon)=1 / u^{1+\epsilon}$, can then be expressed in terms of $z$-space distributions as 


$$
\frac{1}{u^{1+\epsilon}}=-\frac{1-x}{\epsilon} \delta(1-z)+\left[\frac{(1-x / z)^{1+\epsilon}}{(1-z)^{1+\epsilon}}\right]_{\Delta}
$$

The $\Delta$-distribution can be further expanded by $\epsilon$,

$$
\int_{\sqrt{x}}^{1} d z\left[\frac{(1-x / z)^{1+\epsilon}}{(1-z)^{1+\epsilon}}\right]_{\Delta} f(z)=\int_{\sqrt{x}}^{1} d z \frac{1-x / z}{1-z}\left[f(z)-\frac{\left(1-2 x / z+x / z^{2}\right)(1-x)}{(1-x / z)^{2}} f(1)\right]+\mathcal{O}(\epsilon) .
$$

In calculating the Feynman diagrams (c) and (d) in Fig. 2, one encounters the following integral

$$
\int_{0}^{1} d \alpha \frac{\alpha^{-\epsilon}(1-\alpha)^{-\epsilon}}{(1-u) \alpha+u}=\frac{(\Gamma(1-\epsilon))^{2}}{u}{ }_{2} \tilde{F}_{1}\left(1,1-\epsilon ; 2-2 \epsilon ; \frac{u-1}{u}\right),
$$

which is divergent for $u=0$ and $\epsilon=0$. For extraction of $1 / \epsilon$ poles we can use that

$$
\int_{0}^{1} d u u^{-2-\epsilon} \tilde{F}_{1}\left(1,1-\epsilon ; 2-2 \epsilon ; \frac{u-1}{u}\right)=-\frac{1}{4 \epsilon^{3} \Gamma(-2 \epsilon)},
$$

where the regularized hypergeometric function ${ }_{2} \tilde{F}_{1}$ simplifies at $\epsilon=0$ to

$$
{ }_{2} \tilde{F}_{1}\left(1,1 ; 2 ; \frac{u-1}{u}\right)=-\frac{u \ln u}{1-u}
$$

Applying Eq. (A6) then leads to

$$
u^{-2-\epsilon}{ }_{2} \tilde{F}_{1}\left(1,1-\epsilon ; 2-2 \epsilon ; \frac{u-1}{u}\right)=-\frac{1-x}{4 \epsilon^{3} \Gamma(-2 \epsilon)} \delta(1-z)-\left[\frac{(1-x / z)^{2}}{(1-z)(z-x / z)} \ln \frac{(1-z)}{(1-x / z)}\right]_{\Delta}+\mathcal{O}(\epsilon)
$$

\section{APPENDIX B: TWO-LOOP JET AND SOFT FUNCTIONS}

In this Appendix we give the log enhanced two-loop contributions in jet and soft functions, Eqs. (63), (64). First, we consider the moments of the jet function

$$
\tilde{J}[\bar{N}]=\int_{0}^{1} d z z^{-1+N} J\left(Q^{2}(1-z)\right)
$$

In the large $\mathrm{N}$ limit we can write

$$
\tilde{J}[\bar{N}]=1+\frac{\alpha_{s}}{4 \pi}\left(j_{0}^{(1)}+j_{1}^{(1)} L_{j}+j_{2}^{(1)} L_{j}^{2}\right)+\left(\frac{\alpha_{s}}{4 \pi}\right)^{2}\left(j_{1}^{(2)} L_{j}+j_{2}^{(2)} L_{j}^{2}+j_{3}^{(2)} L_{j}^{3}+j_{4}^{(2)} L_{j}^{4}\right),
$$

where $L_{j}=\ln \left(\mu^{2} \bar{N} /\left(M^{2}\left(1-r_{\chi}\right)\right)\right)$ and $\bar{N}=N \exp \left(\gamma_{E}\right)$. Note that in the second line of the equation we dropped the nonlogarithmic term, $\left(\alpha_{s} /(4 \pi)\right)^{2} j_{0}^{(2)}$. The RG equation becomes

$$
\begin{aligned}
\frac{d \tilde{J}}{d \ln \mu}= & \gamma_{J} \tilde{J}=\frac{\alpha_{s}}{4 \pi}\left(\hat{\gamma}_{J, 0}+2 \Gamma_{0} L_{j}\right)+\left(\frac{\alpha_{s}}{4 \pi}\right)^{2}\left[\hat{\gamma}_{J, 1}+\hat{\gamma}_{J, 0} j_{0}^{(1)}+L_{j}\left(\hat{\gamma}_{J, 0} j_{1}^{(1)}+2 \Gamma_{0} j_{0}^{(1)}+2 \Gamma_{1}\right)\right. \\
& \left.+L_{j}^{2}\left(\hat{\gamma}_{J, 0} j_{2}^{(1)}+2 \Gamma_{0} j_{1}^{(1)}\right)+2 \Gamma_{0} j_{2}^{(1)} L_{j}^{3}\right]
\end{aligned}
$$

where we have use that $\gamma_{J}=2 \Gamma_{C} L_{j}+\hat{\gamma}_{J}$, with $\hat{\gamma}_{J}=\sum_{k=0} \hat{\gamma}_{J, k}\left(\alpha_{S} /(4 \pi)\right)^{k}$. From this we can obtain the coefficients of the $\alpha_{s}^{2} \log$ terms in Eq. (B2),

$$
j_{1}^{(2)}=\frac{1}{2}\left(\hat{\gamma}_{J, 1}+\hat{\gamma}_{J, 0} j_{0}^{(1)}+2 \beta_{0} j_{0}^{(1)}\right),
$$




$$
\begin{gathered}
j_{2}^{(2)}=\frac{1}{4}\left(\left(\hat{\gamma}_{J, 0}+2 \beta_{0}\right) j_{1}^{(1)}+2 \Gamma_{0} j_{0}^{(1)}+2 \Gamma_{1}\right), \\
j_{3}^{(2)}=\frac{1}{6}\left(\left(\hat{\gamma}_{J, 0}+2 \beta_{0}\right) j_{2}^{(1)}+2 \Gamma_{0} j_{1}^{(1)}\right), \\
j_{4}^{(2)}=\frac{1}{8}\left(2 \Gamma_{0} j_{2}^{(1)}\right) .
\end{gathered}
$$

In a similar way, we write for the soft function

$$
\begin{aligned}
\tilde{S}[\bar{N}]= & 1+\frac{\alpha_{s}}{4 \pi}\left(s_{0}^{(1)}+s_{1}^{(1)} L_{s}+s_{2}^{(1)} L_{s}^{2}\right) \\
& +\left(\frac{\alpha_{s}}{4 \pi}\right)^{2}\left(s_{1}^{(2)} L_{s}+s_{2}^{(2)} L_{s}^{2}+s_{3}^{(2)} L_{s}^{3}+s_{4}^{(2)} L_{s}^{4}\right),
\end{aligned}
$$

where $L_{s}=\ln \left(\mu^{2} \bar{N} / M^{2}\right)$, and the coefficients of the $\alpha_{s}^{2} \log$ terms are determined to be

$$
\begin{gathered}
s_{1}^{(2)}=\frac{1}{2}\left(\hat{\gamma}_{s, 1}+\left(\hat{\gamma}_{s, 0}+2 \beta_{0}\right) s_{0}^{(1)}\right), \\
s_{2}^{(2)}=\frac{1}{4}\left(\left(\hat{\gamma}_{s, 0}+2 \beta_{0}\right) s_{1}^{(1)}+A_{s}\left(\Gamma_{0} s_{0}^{(1)}+\Gamma_{1}\right)\right), \\
s_{3}^{(2)}=\frac{1}{6}\left(\left(\hat{\gamma}_{s, 0}+2 \beta_{0}\right) s_{2}^{(1)}+A_{s} \Gamma_{0} s_{1}^{(1)}\right), \\
s_{4}^{(2)}=\frac{1}{8}\left(A_{s} \Gamma_{0} s_{2}^{(1)}\right),
\end{gathered}
$$

where $A_{s}=-1, \quad \gamma_{S}=A_{s} \Gamma_{C} L_{s}+\hat{\gamma}_{S}, \quad$ and $\quad \hat{\gamma}_{S}=$ $\sum_{k=0} \hat{\gamma}_{S, k}\left(\alpha_{s} /(4 \pi)\right)^{k}$.
[1] G. Jungman, M. Kamionkowski, and K. Griest, Supersymmetric dark matter, Phys. Rep. 267, 195 (1996).

[2] G. Steigman and M. S. Turner, Cosmological constraints on the properties of weakly interacting massive particles, Nucl. Phys. B 253, 375 (1985).

[3] B. W. Lee and S. Weinberg, Cosmological Lower Bound on Heavy Neutrino Masses, Phys. Rev. Lett. 39, 165 (1977).

[4] G. Bertone, D. Hooper, and J. Silk, Particle dark matter: Evidence, candidates and constraints, Phys. Rep. 405, 279 (2005).

[5] A. J. Barr and C. G. Lester, A review of the mass measurement techniques proposed for the Large Hadron Collider, J. Phys. G 37, 123001 (2010).

[6] A. J. Barr, T. J. Khoo, P. Konar, K. Kong, C. G. Lester, K. T. Matchev, and M. Park, Guide to transverse projections and mass-constraining variables, Phys. Rev. D 84, 095031 (2011).

[7] M. Burns, K. Kong, K. T. Matchev, and M. Park, Using subsystem MT2 for complete mass determinations in decay chains with missing energy at hadron colliders, J. High Energy Phys. 03 (2009) 143.

[8] P. Konar, K. Kong, K. T. Matchev, and M. Park, Dark matter particle spectroscopy at the LHC: Generalizing M(T2) to asymmetric event topologies, J. High Energy Phys. 04 (2010) 086.

[9] P. Konar, K. Kong, K. T. Matchev, and M. Park, Superpartner Mass Measurement Technique using 1D Orthogonal Decompositions of the Cambridge Transverse Mass Variable $M_{T 2}$, Phys. Rev. Lett. 105, 051802 (2010).

[10] W. S. Cho, K. Choi, Y. G. Kim, and C. B. Park, Gluino Stransverse Mass, Phys. Rev. Lett. 100, 171801 (2008).

[11] W. S. Cho, K. Choi, Y. G. Kim, and C. B. Park, Measuring superparticle masses at hadron collider using the transverse mass kink, J. High Energy Phys. 02 (2008) 035.

[12] D. Kim, K. T. Matchev, F. Moortgat, and L. Pape, Testing invisible momentum Ansatze in missing energy events at the LHC, J. High Energy Phys. 08 (2017) 102.
[13] D. Debnath, D. Kim, J.H. Kim, K. Kong, and K. T. Matchev, Resolving combinatorial ambiguities in Dilepton $t \bar{t}$ event topologies with constrained $M_{2}$ variables, Phys. Rev. D 96, 076005 (2017).

[14] K. J. Bae, T. H. Jung, and M. Park, Spectral Decomposition of Missing Transverse Energy at Hadron Colliders, Phys. Rev. Lett. 119, 261801 (2017).

[15] K. Kawagoe, M. M. Nojiri, and G. Polesello, A new SUSY mass reconstruction method at the CERN LHC, Phys. Rev. D 71, 035008 (2005).

[16] M. M. Nojiri, G. Polesello, and D. R. Tovey, A hybrid method for determining SUSY particle masses at the LHC with fully identified cascade decays, J. High Energy Phys. 05 (2008) 014.

[17] H.-C. Cheng, D. Engelhardt, J. F. Gunion, Z. Han, and B. McElrath, Accurate Mass Determinations in Decay Chains with Missing Energy, Phys. Rev. Lett. 100, 252001 (2008).

[18] W. S. Cho, K. Choi, Y. G. Kim, and C. B. Park, M(T2)assisted on-shell reconstruction of missing momenta and its application to spin measurement at the LHC, Phys. Rev. D 79, 031701 (2009).

[19] S. P. Martin, A Supersymmetry Primer, Advanced Series on Directions in High Energy Physics Vol. 18, pp. 1-98. (1997), https://doi.org/10.1142/9789812839657_0001.

[20] M. Beneke, L. Jenniches, A. Mck, and M. Ubiali, Radiative distortion of kinematic edges in cascade decays, Phys. Lett. B 770, 539 (2017).

[21] R. Horsky, M. Krmer, A. Muck, and P. M. Zerwas, Squark cascade decays to Charginos/Neutralinos: Gluon radiation, Phys. Rev. D 78, 035004 (2008).

[22] W. Hollik, J. M. Lindert, and D. Pagani, NLO corrections to squark-squark production and decay at the LHC, J. High Energy Phys. 03 (2013) 139.

[23] W. Hollik, J. M. Lindert, and D. Pagani, On cascade decays of squarks at the LHC in NLO QCD, Eur. Phys. J. C 73, 2410 (2013). 
[24] C. W. Bauer, S. Fleming, and M. E. Luke, Summing Sudakov logarithms in $\mathrm{B} \rightarrow X$ (s gamma) in effective field theory, Phys. Rev. D 63, 014006 (2000).

[25] C. W. Bauer, S. Fleming, D. Pirjol, and I. W. Stewart, An effective field theory for collinear and soft gluons: Heavy to light decays, Phys. Rev. D 63, 114020 (2001).

[26] C. W. Bauer, D. Pirjol, and I. W. Stewart, Soft collinear factorization in effective field theory, Phys. Rev. D 65, 054022 (2002).

[27] S. W. Bosch, B. O. Lange, M. Neubert, and G. Paz, Factorization and shape function effects in inclusive B meson decays, Nucl. Phys. B 699, 335 (2004).

[28] C. Kim, A. Idilbi, T. Mehen, and Y. W. Yoon, Production of stoponium at the LHC, Phys. Rev. D 89, 075010 (2014).

[29] M. Beneke, J. Piclum, C. Schwinn, and C. Wever, NNLL soft and Coulomb resummation for squark and gluino production at the LHC, J. High Energy Phys. 10 (2016) 054.

[30] M. Drees and M. M. Nojiri, Production and decay of scalar stoponium bound states, Phys. Rev. D 49, 4595 (1994).

[31] S. P. Martin, Diphoton decays of stoponium at the Large Hadron Collider, Phys. Rev. D 77, 075002 (2008).

[32] B. Batell and S. Jung, Probing light stops with stoponium, J. High Energy Phys. 07 (2015) 061.

[33] J. Chay, C. Kim, Y. G. Kim, and J.-P. Lee, Soft Wilson lines in soft-collinear effective theory, Phys. Rev. D 71, 056001 (2005).

[34] C. W. Bauer and A. V. Manohar, Shape function effects in $B \rightarrow X_{s} \gamma$ and $B \rightarrow X_{u} l \bar{\nu}$ decays, Phys. Rev. D 70, 034024 (2004).

[35] G. P. Korchemsky and A. V. Radyushkin, Renormalization of the Wilson loops beyond the leading order, Nucl. Phys. B 283, 342 (1987).

[36] I. A. Korchemskaya and G. P. Korchemsky, On lightlike Wilson loops, Phys. Lett. B 287, 169 (1992).

[37] S. Moch, J. A. M. Vermaseren, and A. Vogt, The three loop splitting functions in QCD: The Nonsinglet case, Nucl. Phys. B 688, 101 (2004).

[38] M. Neubert, Renormalization-group improved calculation of the $\mathrm{B} \rightarrow X(\mathrm{~s})$ gamma branching ratio, Eur. Phys. J. C 40, 165 (2005).

[39] M. Neubert, Advanced predictions for moments of the $\bar{B} \rightarrow X_{s} \gamma$ photon spectrum, Phys. Rev. D 72, 074025 (2005).

[40] T. Becher and M. Neubert, Threshold Resummation in Momentum Space from Effective Field Theory, Phys. Rev. Lett. 97, 082001 (2006).

[41] T. Becher, M. Neubert, and B. D. Pecjak, Factorization and momentum-space resummation in deep-inelastic scattering, J. High Energy Phys. 01 (2007) 076.

[42] L. G. Almeida, S. D. Ellis, C. Lee, G. Sterman, I. Sung, and J. R. Walsh, Comparing and counting logs in direct and effective methods of QCD resummation, J. High Energy Phys. 04 (2014) 174.

[43] R. W. Assmann, A 3-TeV $e^{+} e^{-}$Linear Collider based on CLIC technology, CERN Report No. CERN-2000-008, 2000.

[44] E. Accomando et al. (CLIC Physics Working Group), Physics at the CLIC multi-TeV linear collider, Report No. (2004), https://doi.org/10.5170/CERN-2004-005.
[45] M. Aicheler, P. Burrows, M. Draper, T. Garvey, P. Lebrun, K. Peach, N. Phinney, H. Schmickler, D. Schulte, and N. Toge, A Multi-TeV Linear Collider Based on CLIC Technology (2012), https://doi.org/10.5170/CERN-2012-007.

[46] H. Abramowicz et al., Higgs physics at the CLIC electronpositron linear collider, Eur. Phys. J. C 77, 475 (2017).

[47] D. Dannheim, P. Lebrun, L. Linssen, D. Schulte, F. Simon, S. Stapnes, N. Toge, H. Weerts, and J. Wells, CLIC e+elinear collider studies arXiv:1208.1402.

[48] M. Battaglia, J.-J. Blaising, J. S. Marshall, S. Poss, A. Sailer, M. Thomson, and E. van der Kraaij, Physics performance for scalar electron, scalar muon and scalar neutrino searches at $\sqrt{s}=3 \mathrm{TeV}$ and $1.4 \mathrm{TeV}$ at CLIC, J. High Energy Phys. 09 (2013) 001.

[49] A. Munnich, Measurement of $\tilde{\tau_{1}}$ pair production at CLIC, LCD-Note-2012-004.

[50] P. Roloff, T. Barklow, and A. Munnich, Measurement of chargino and neutralino pair production at CLIC, LCDNote-2011-037.

[51] F. Simon and L. Weuste, Light-flavor squark reconstruction at CLIC, Eur. Phys. J. C 75, 379 (2015).

[52] D. R. Tovey, On measuring the masses of pair-produced semi-invisibly decaying particles at hadron colliders, J. High Energy Phys. 04 (2008) 034.

[53] J. Alwall, M. Herquet, F. Maltoni, O. Mattelaer, and T. Stelzer, MadGraph 5: Going beyond, J. High Energy Phys. 06 (2011) 128.

[54] J. Alwall, R. Frederix, S. Frixione, V. Hirschi, F. Maltoni, O. Mattelaer, H. S. Shao, T. Stelzer, P. Torrielli, and M. Zaro, The automated computation of tree-level and next-to-leading order differential cross sections, and their matching to parton shower simulations, J. High Energy Phys. 07 (2014) 079.

[55] T. Sjostrand, S. Mrenna, and P.Z. Skands, PYTHIA 6.4 physics and manual, J. High Energy Phys. 05 (2006) 026.

[56] N. D. Christensen, P. de Aquino, C. Degrande, C. Duhr, B. Fuks, M. Herquet, F. Maltoni, and S. Schumann, A comprehensive approach to new physics simulations, Eur. Phys. J. C 71, 1541 (2011).

[57] C. Duhr and B. Fuks, A superspace module for the FeynRules package, Comput. Phys. Commun. 182, 2404 (2011).

[58] M. Cacciari, G. P. Salam, and G. Soyez, FastJet user manual, Eur. Phys. J. C 72, 1896 (2012).

[59] M. Cacciari, G. P. Salam, and G. Soyez, The Anti-k(t) jet clustering algorithm, J. High Energy Phys. 04 (2008) 063.

[60] J. L. Feng and D. E. Finnell, Squark mass determination at the next generation of linear $e^{+} e^{-}$colliders, Phys. Rev. D 49, 2369 (1994).

[61] F. Simon, Techniques and prospects for light-flavored sQuark mass measurements at a multi- $\mathrm{TeV} e^{+} e^{-}$Collider, Report No. LCD-Note-2010-012 2010, http://cds.cern.ch/record/ 1443546.

[62] A. K. Datta, K. Kong, and K. T. Matchev, The impact of beamstrahlung on precision measurements at CLIC, eConf C050318, 0215 (2005), arXiv:hep-ph/0508161.

[63] D. Schulte, High-energy beam-beam effects in CLIC, Report No. CERN-PS-99-017-LP, CERN-PS-99-17-LP, CERN-CLIC-NOTE-391, CLIC-NOTE-391 1999. 